Aus der Heilstätte Holsterhausen bei Werden a. d. Ruhr.

(Chefarzt Dr. F. Köhler.)

\title{
Die Affektionen der oberen Luftwege bei Phthisikern in den Anfangsstadien.
}

\author{
Von
}

Dr. med. Max Behr,

Assistenzarzt der Königl. laryngolog. Poliklinik der Universität Kiel, früherem Hausarzte der Heilstüte Holsterhauson bei Werden a. d. Ruhr.

„Phthisis laryngea tuberculosa!" Hätten wir keine anderen Anhaltspunkte, so würde schon dieser Name uns zeigen, welche Wandlungen der klinisch-pathologische Begriff dieses Leidens bis zu unserer heutigen Anschauung durchgemacht haben muss. Das letzte der drei Worte setzen wir in unserer heutigen Terminologie an die erste Stelle; das Adjektivum wird von uns zum "Hanptwort" befördert, und ihm die Lokalbezeichnung beigegeben: "Tuberculosis laryngea", sagen wir und heben die Art der Erkrankung als das Wichtigste für ihre Bekämpfung hervor. Die Phthisis, welche den alten Ärzten das Merkmal war, möchten wir nach Möglichkeit verhüten, da sie leider mehr der Gegenstand unseres Mitleides als einer erfolgreichen $\mathrm{Be}-$ handlung sein kann.

Dieses Streben nach möglichst frülızeitiger Erkennung der spezifischen Art der Affektionen hat begreitlicherweise mit der Verfeinerung unserer diagnostischen Hilfsmittel auch zu zahlreichen Meinungsverschiedenheiten geführt, bei welchen wir in vielen Fällen von einer Einigung noch weit entfernt sind.

Gerade jetzt, wo die Tuberkulose in aller Munde und der Mittelpunkt des Interesses ist, wo aber auch der Kriegsruf in den gegnerischen Lagern widerhallt, und die Kampfeswogen hoch gehen wie nie zuvor, jetzt dürfte wohl jeder Beitrag zur Stütze des einen oder anderen Standpunktes von Wert sein.

So möchte ich an der Hand des mir von meinem sehr verehrten Chef in dankenswerter Weise bereitwilligst überlassenen Materials einige Beobachtungen mitteilen, welche dentlich die grosse Zahl der Erkrankungen der oberen Luftwege bei den Phthisikern dartun und meines Erachtens unbedingt für einen Zusammenhang dieser Affek- 
tionen ron Nase, Hals und Kehlkopf sprechen. Trotz der gerade in den letzten Jahren von H. Schmidt, Loeri, Friedrich, Blumenfeld, Möller, Beckmann, Freudenthal u. a. immer wieder hervorgehobenen Beziehungen zwischen Lungenerkrankungen und pathologischen Veränderungen der oberen Luftwege wird gewiss der Atmungstraktus vielfach noch zu wenig als zusammengehöriges Ganzes betrachtet, und die einzelnen Abweichungen vom gesunden Zustande noch nicht genug im Rahmen der übrigen Erkrankungen als unzertrennlich gewürdigt.

Möller und Rappoport (Zeitschrift für Tuberkulose und Heilstättenwesen 1903, Band IV, Heft 5) haben vor zwei Jahren an 120 Patienten die „Beziehungen der nicht tuberkulösen Erkrankungen der oberen Luftwege zur Lungentuberkulose " untersucht und in $84^{\%} \%$ die Nase, in $76 \%$ den Rachen, in $42 \%$ den Kehlkopf erkrankt gefunden; die Arbeiten von W. Freudenthal u. a. zeigen gleichfalls die grosse Häufigkeit jener korrespondierenden krankhaften Veränderungen. Wie oft aber dieselben als tuberkulös $\mathrm{zu}$ bezeichnen sind und wie oft nicht, darüber gehen die Ansichten der einzelnen Autoren weit auseinander; schwanken doch z. B. die angegebenen Zahlen für die Mitbeteiligung des Larynx bei Lungentuberkulose zwischen $13 \%$ (Willigk) und $97 \%$ (Schaefer)!

Dass der Phthisiker oder der zur Tuberkulose Disponierte leichter zu Erkrankungen der oberen Luftwege neigt, dürfte wohl zweifellos sein, sicherlich $\mathrm{zu}$ den akuten katarrhalischen, schon wegen seiner grossen Empfindlichkeit gegen Erkältungen. Aber auch die chronischen und dauernden Veränderungen treffen wir bei ihnen häufiger an als bei Nichttuberkulösen. Ich möchte glauben, dass eine Reihe von ihnen den veränderten Verhältnissen von Nase, Racheu und Kehlkopf ihr Lungenleiden mehr oder weniger zu verdanken haben, und Untersuchungen der neuesten Zeit (A ufrecht, Beckmann, Frese, Westenhöffer u. a.) sprechen wahrlich dafür.

Die oberen Luftwege, insbesondere die Nase, wirken gewissermassen präparator is ch auf die Atmungsluft. Diese wird zunächst bis zu einem gewissen Grade gereinigt, einmal von anorganischen Beimischungen, dann auch von organischen, vor allem Bakterien, unter welchen von Möller und Rappoport der Staphylococcus pyogenes aureus, der Friedländer sche Pneumoniebacillus, der Streptococcus, der Fränkel-Weichselbaumsche Diplococcus, der Tuberkelbacillus u. a. m. angeführt werden. Ferner findet eine Erwärmung der Inspirationsluft statt, und zwar wie die Untersuchungen von Aschenbrandt und $K$ ayser (Pflügers Arch. Bd. 41) ergeben haben, auf $30^{\circ} \mathrm{C}$, einerlei wie die Temperatur der Aussenluft ist. 
An der dritten Funktion der Nase, der Anfeuchtung der Luft, ist nach Freudenthal (Beiträge zur Klinik der Tuberkulose 1903, Bd. II, Heft 1) der Nasenrachenraum stark beteiligt.

Wird diese Tätigkeit ausgeschaltet, so stellen sich bald krankhafte Veränderungen infolge ungenügender Vorbereitung der Atmungsluft ein, die auch per continuitatem aut die tieferen Teile des Atmungstraktus übergehen, und die Ursachen von Entzündungserscheinungen. Infektionskrankheiten etc. abgeben.

In recht übersichtlicher und klarer Weise schildert E. P. Friedrich (Rhinologie, Laryngologie und Otologie in ihrer Bedeutung für die allgemeine Medizin, 1899, F. C. W. Vogel-Leipzig) diese Beziehungen der einzelnen Abschnitte des Atmungstraktus und ihrer pathologischen Veränderungen zu einander. Die durch die „Störung der Funktionen der oberen Luftwege" bedingte Mundatmung führt zu den chronisch katarrhalischen Zuständen der oberen und tieferen Luftwege und bedingt eine grosse Disposition für Katarrhe des Pharynx, des Larynx und der Bronchien. Doch nicht allein sekundär und deszendierend schreiten die Erkrankungen fort; Sticker (Deutsch. Arch. f. klin. Med. Bd. 57) nimmt eine allgemeine Xer ose an, welche die gesamten Schleimhäute des Körpers befallen und zur Atrophie führen kann, und vieles spricht für diese Ansicht. Wir hätten somit die gleiche Ursache für die Erkrankungen der Atmungsorgane als Ganzes. Auch Freudenthal scheint dieser Ansicht, obwohl er die Beteiligung der Lunge nicht sonderlich hervorhebt, nicht fern zu stehen; er benennt den Zustand Xerasie. Schliesslich sei noch die auch von Friedrich hervorgehobene aszendierende Form erwähnt, die von den unteren Abschnitten aus auf die oberen übergeht.

Bevor ich auf die einzelnen Erkrankungen, welche uns bei unseren Beobachtungen als die häufigsten imponierten, eingehe, möchte ich in der folgenden Übersicht die pathologischen Veränderụngen von 300 Kranken mitteilen. Die Absicht, möglichst objektiv die Beziehungen der Affektionen der oberen Luftwege zueinander und zur Lungentuberkulose beurteilen zu können, liess mich die Untersuchungen in der Form der Eintagstatistik vurnehmen, da auf diese Weise bei Heranziehung aller Heilstätteninsassen eine Auswahl des Krankenmaterials ausgeschlossen ist. Der Untersuchungsbefund wurde jedesmal nach meinem Diktate aufgezeichnet.

\section{Statistischer Teil.}

In einem vor wenigen Monaten veröffentlichten Aufsatze macht Frese (Wünch. med. Wochenschrift 1904, Nr. 13) für das Zustandekommen der verschiedenartigen Ergebnisse bei statistischen Erhe- 
bungen die ungleiche Genauigkeit der Untersuchung, die verschiedene Art des Krankenmaterials und die nicht übereinstimmende Deutung des Befundes verantwortlich. Ferner scheinen ihm die an Lebenden gewonnenen Zahlen für die Miterkrankung des Kehlkopfes bei Lungentuberkulose niedriger sein zu müssen als die an Leichenmaterial festgestellten; „denn jeder Untersucher sieht auch initiale Lungenphthisen, bei denen Kehlkopfveränderungen seltener sind."

Zur richcigen Beurteilung der bei unserer Untersuchungsreihe gefundenen Zahlen scheint es mir erforderlich, in Kürze auf jene Punkte einzugehen.

1. Was die Art der Untersuchung angeht, so wurden nur die als wirklich krankhaft imponierenden und durch objektive Symptome sich manifestierenden Veränderungen der oberen Luftwege diagnostiziert und aufgezeichnet. Minimale Abweichungen z. B. im Bau der Nase, des Rachens etc., die weder subjektive Beschwerden noch anderweitige Zeichen eines pathologischen Zustandes zeitigten, wurden nicht berücksichtigt. Ich glaube dies hervorheben zu müssen, da man wohl auch bei "Gesunden" durch eine allzufeine Diagnostik es erreichen kann, z. B. nur einen minimalen Prozentsatz „normaler" Nasen zu erhalten.

2. Unser Krankenmaterial ist ziemlich eindeutig; es sind alles Kranke, bei welchen ein wirtschaftlicher Erfolg voraussichtlich erreicht wird, die sich also klinisch sozusagen im Anfangsstadium der Tuberkulose befinden.

3. Über die Deutung des Befundes herrscht allgemein wenig Einigkeit. Ich habe schon in der Einleitung als Beispiel die ungeheure Schwankung des Prozentsatzes der Larynxphthise bei den verschiedenen Autoren erwähnt. „Wer jede Parese und leichte Schleimbautrötung schon als Ausdruck einer spezifischen Erkrankung ansieht, wird natürlich zu sehr hohen Zahlen kommen." (Frese.) Welcher Standpunkt der richtige ist, wage ich nicht zu entscheiden. Jedenfalls scheint es mir sicher, dass der Phthisiker nicht nur in gleicher Weise wie jeder andere, sondern noch weit mehr auch zu katarrhalischen Erkrankungen des Halses wie der übrigen Schleimhäute infolge seiner grossen Empfindlichkeit und Verweichlichung neigt. Ich möchte daher nicht jeden Ausdruck einer entzündlichen Reaktion im Kehlkopfe als ein unbedingtes Symptom einer bestehenden lokalen Phthise verwerten. Ich habe auch in meiner Statistik nur solche Befunde mit "Tbc." belegt, welche mir unzweifelhaft als spezifisch erschienen.

Wie jede Statistik, so beansprucht auch die unserige nach alledem nur relativen Wert. Subjektive Momente werden sich nie ausschalten lassen und bei der Beurteilung eines summarisch aufgestellten grösseren Krankenmaterials gewisse Rücksicht erheischen. 
5] Die Affektionen d. oberen Luftwege bei Phthisikern in den Anfangsstadien. 41

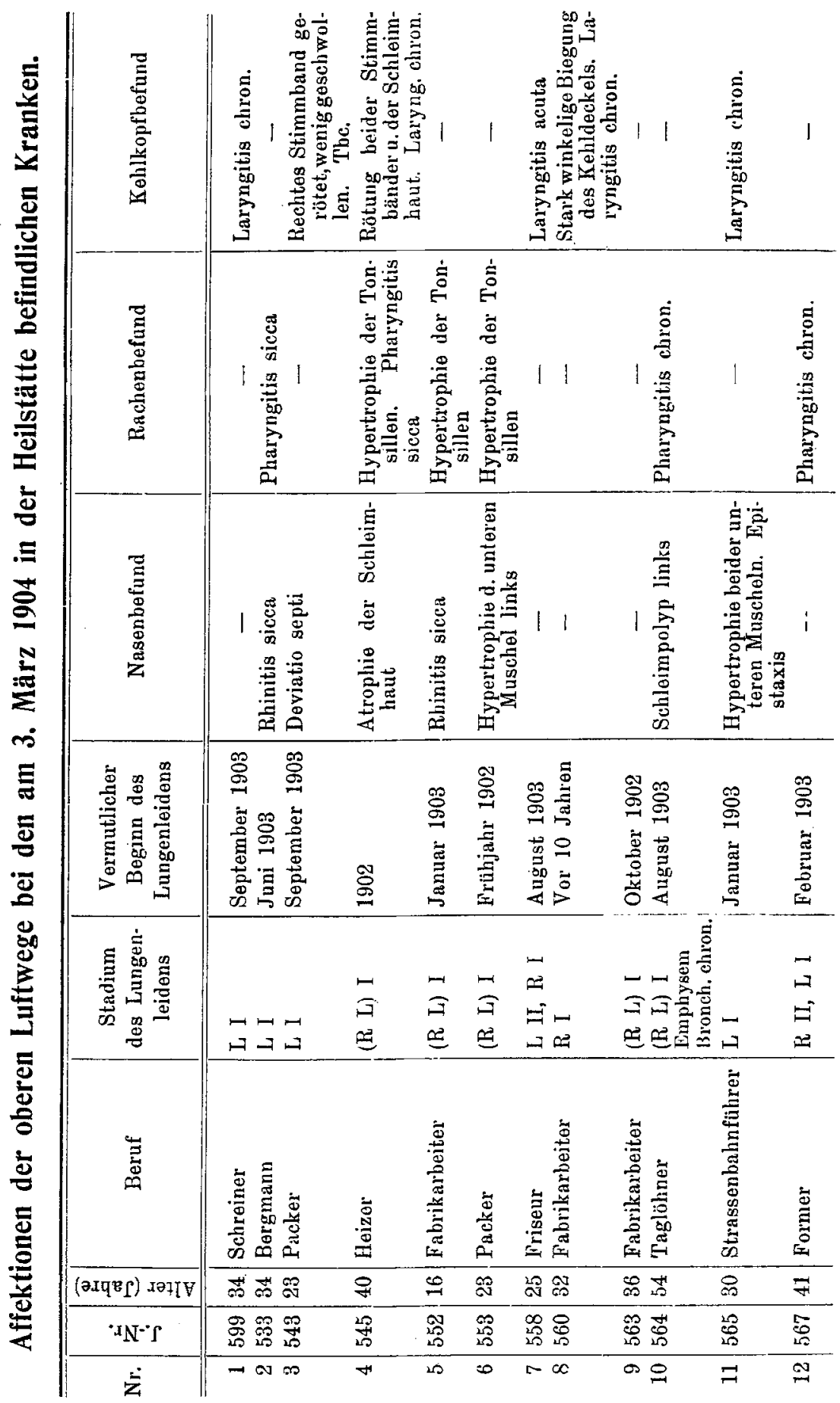




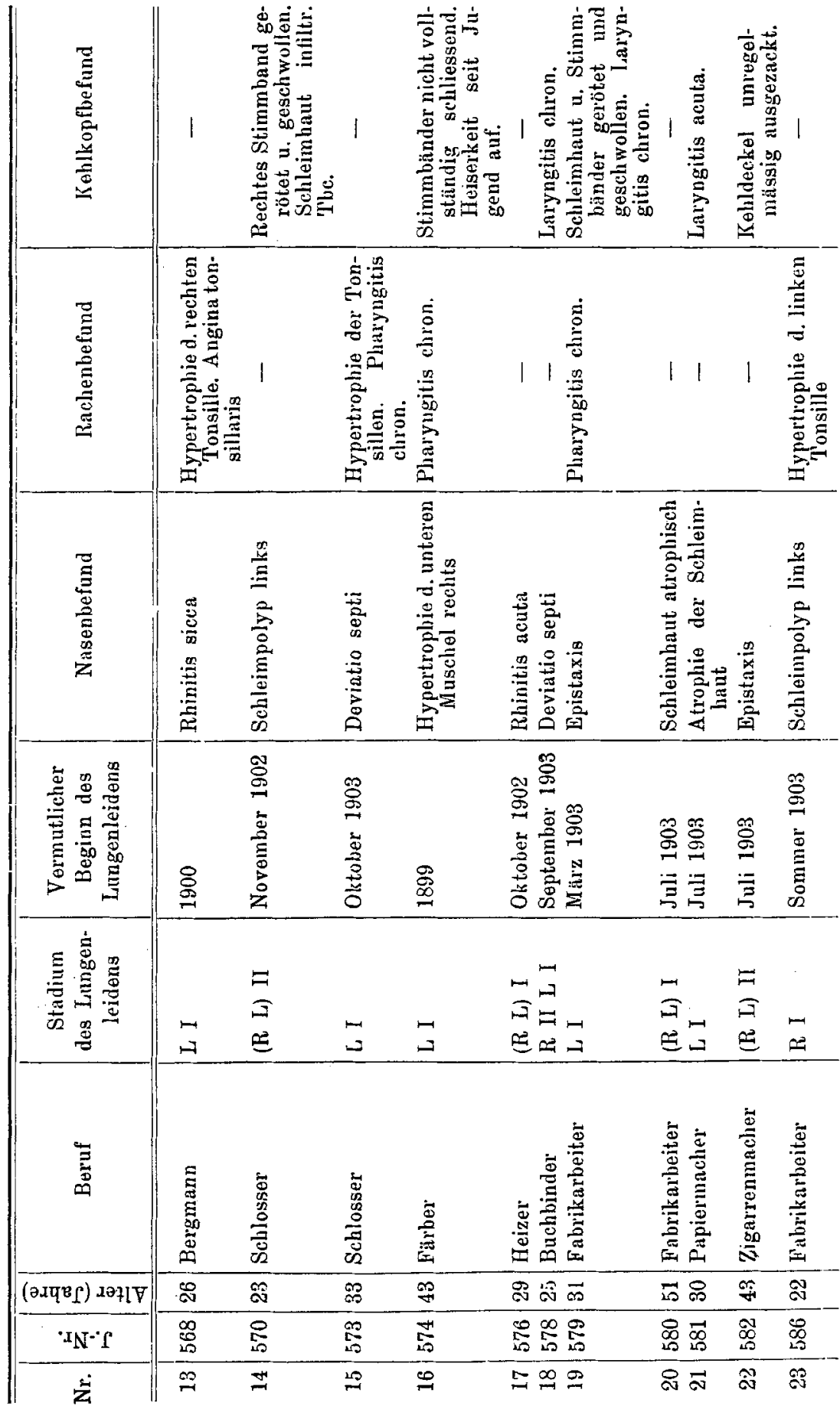


7] Die Affeltionen d. oberen Luftwege bei- Phthisikern in den Anfangsstadien. 43

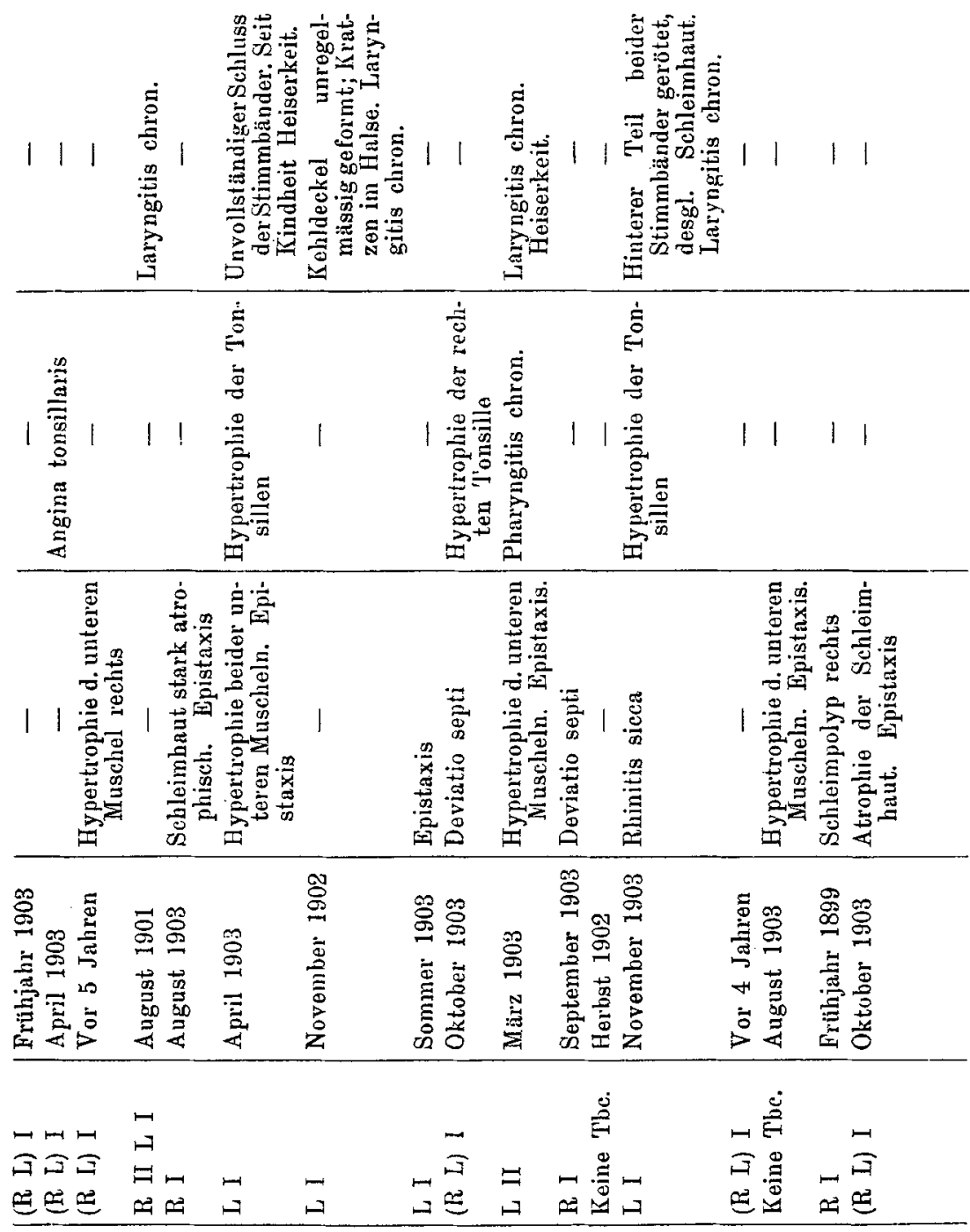

\begin{tabular}{|c|c|c|c|c|c|c|c|c|}
\hline 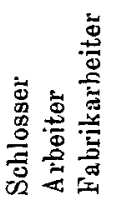 & 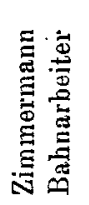 & 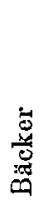 & 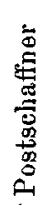 & 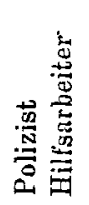 & 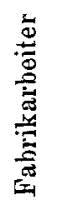 & 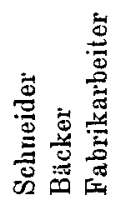 & 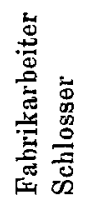 & 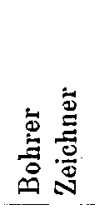 \\
\hline 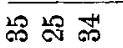 & $\stackrel{\circ}{9}$ & $\stackrel{\circ}{\circ}$ & $\mathscr{m}$ & 음 & $\overline{\mathfrak{g}}$ & 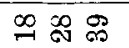 & 令 尺ิ & 웅 \\
\hline 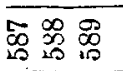 & 扂孚 & 落 & $\mathscr{9}$ & 58 & 5 & 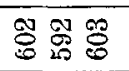 & \% & 8 \\
\hline ล̊ำ & $\Sigma \infty$ & ని & 요 & का ติ & $\stackrel{m}{\infty}$ & 車 & 乩象 & 용 \\
\hline
\end{tabular}




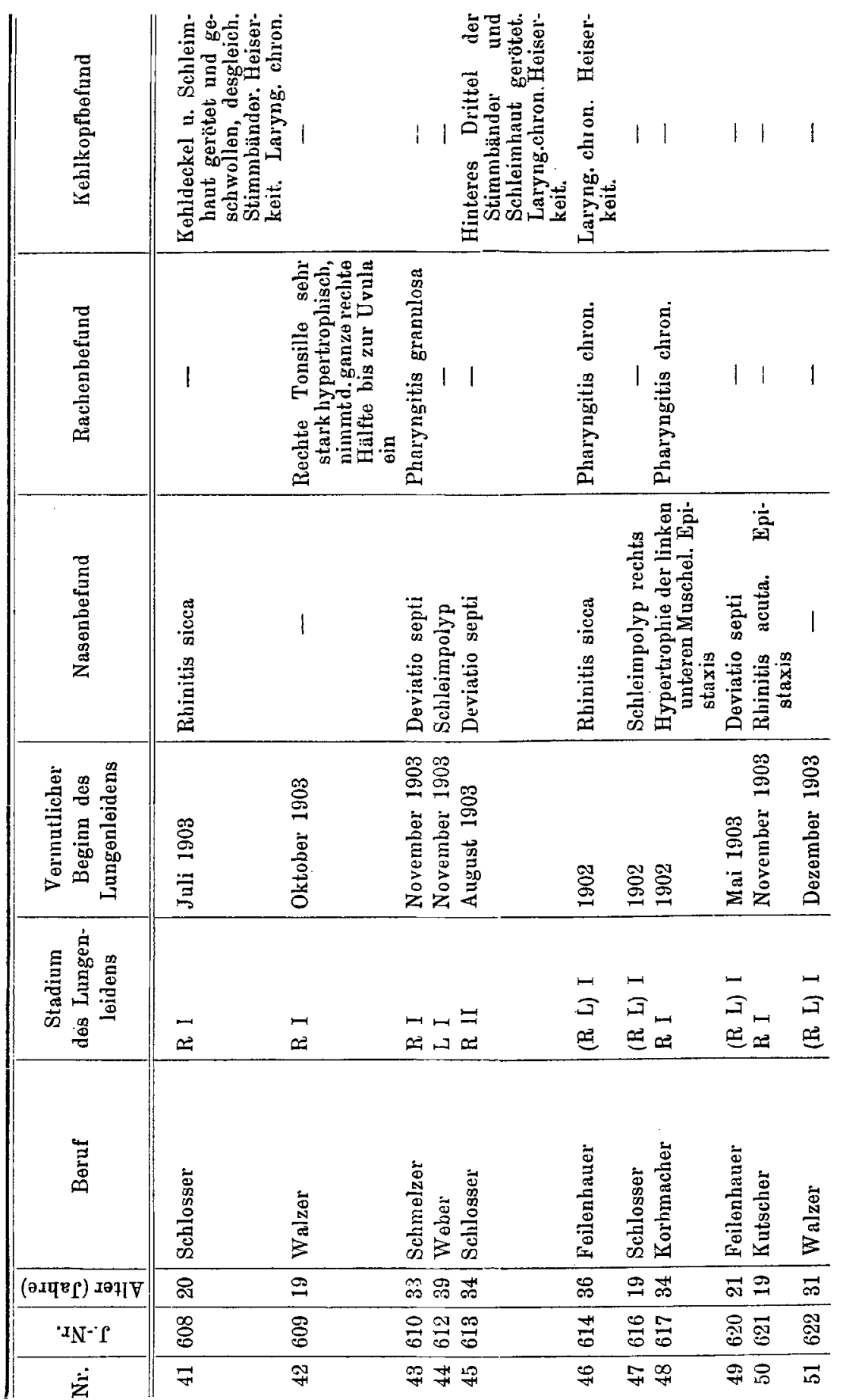


9] Die Affektionen d. oberen Luftwege bei Phthisikern in den Anfangsstadien.
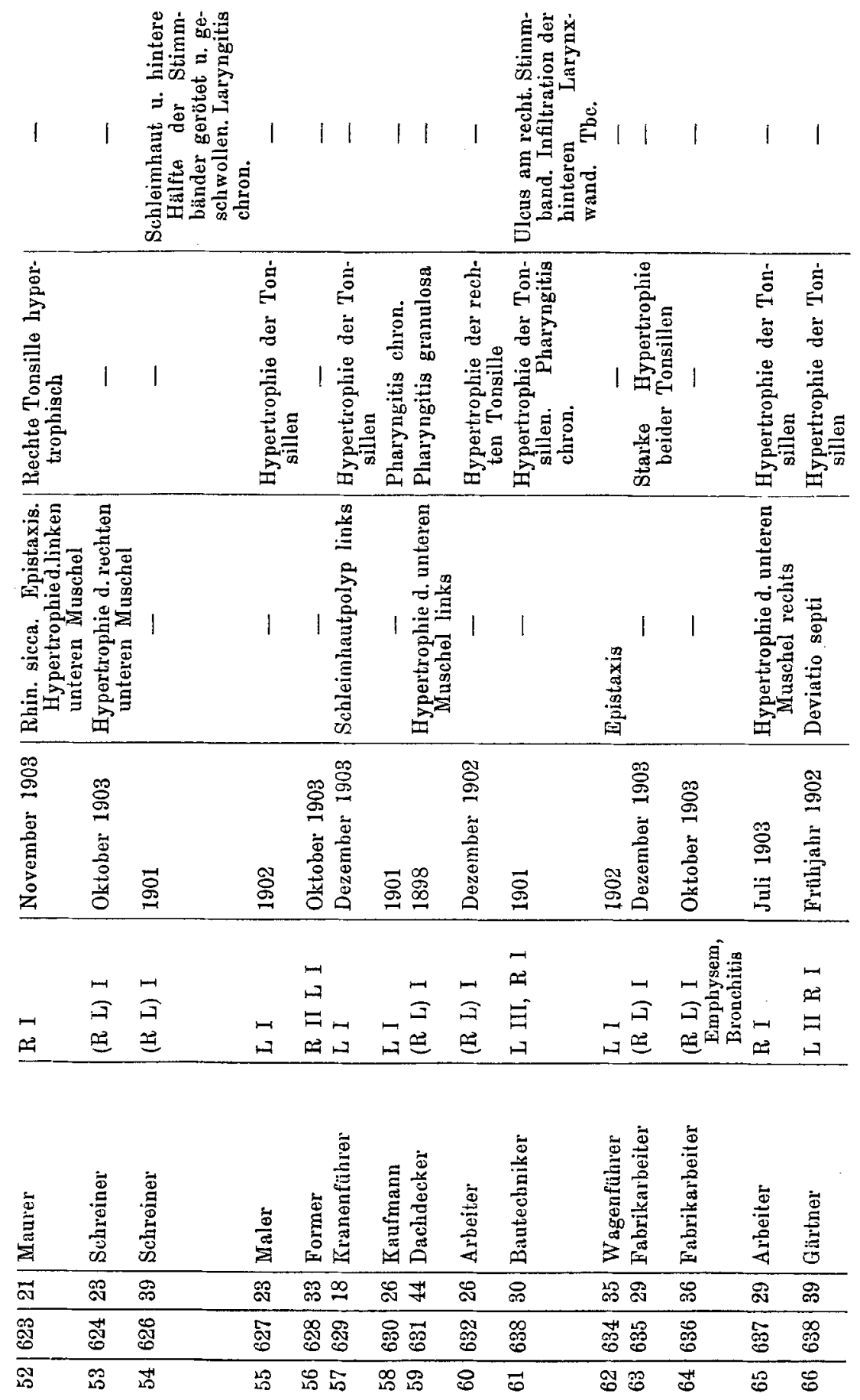


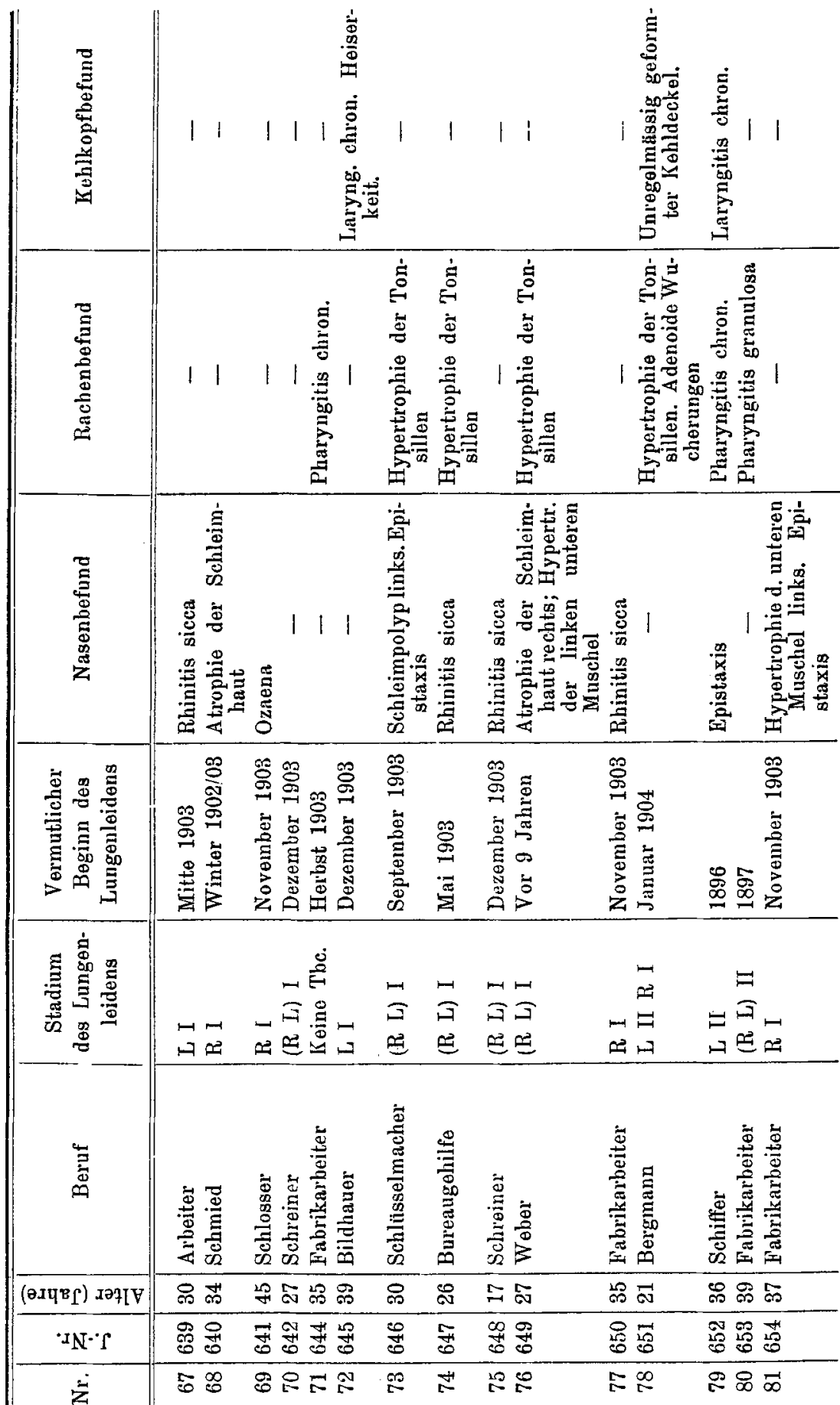


11] Die Affektionen d. oberen Luftwege bei Phthisikern in den Anfangsstadien. 47
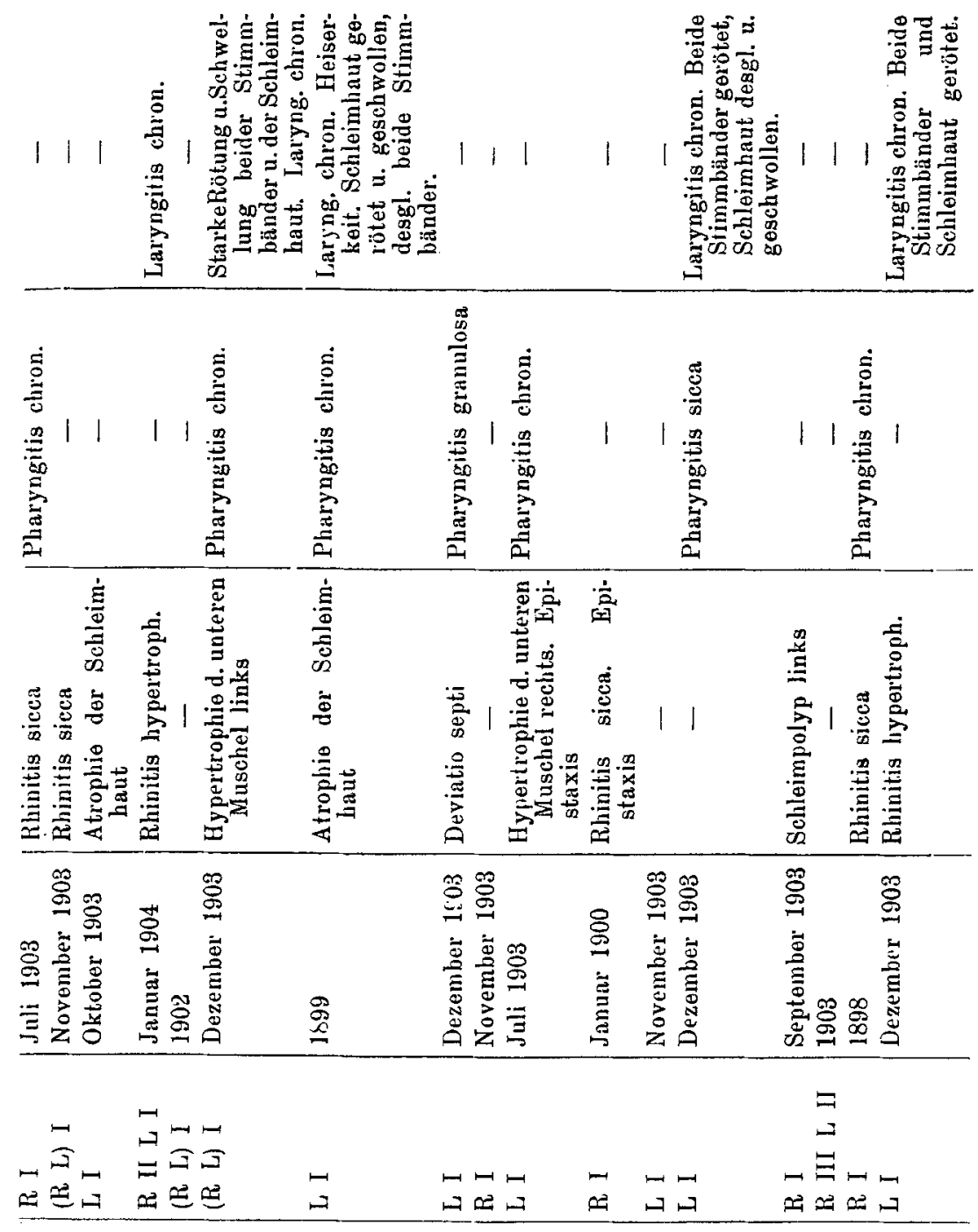

\begin{tabular}{|c|c|c|c|c|c|c|}
\hline 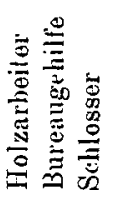 & 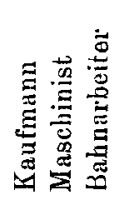 & 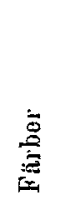 & 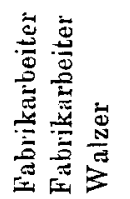 & 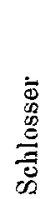 & 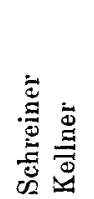 & 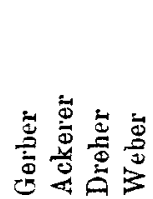 \\
\hline 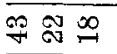 & 욤 & $\widehat{s}$ & 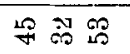 & $\dddot{m}$ & $\widehat{\mathrm{s}}$ & 雨 次 \\
\hline 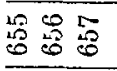 & $\begin{array}{l}508 \\
108 \\
0\end{array}$ & $\overrightarrow{0}$ & 丹̊ & 80 & $\mathscr{8} 8$ & 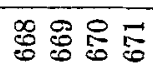 \\
\hline 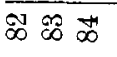 & $\infty \infty \infty$ & 舟 & ब 8 & $\stackrel{\sigma}{\sigma}$ & ळ & 19 \\
\hline
\end{tabular}




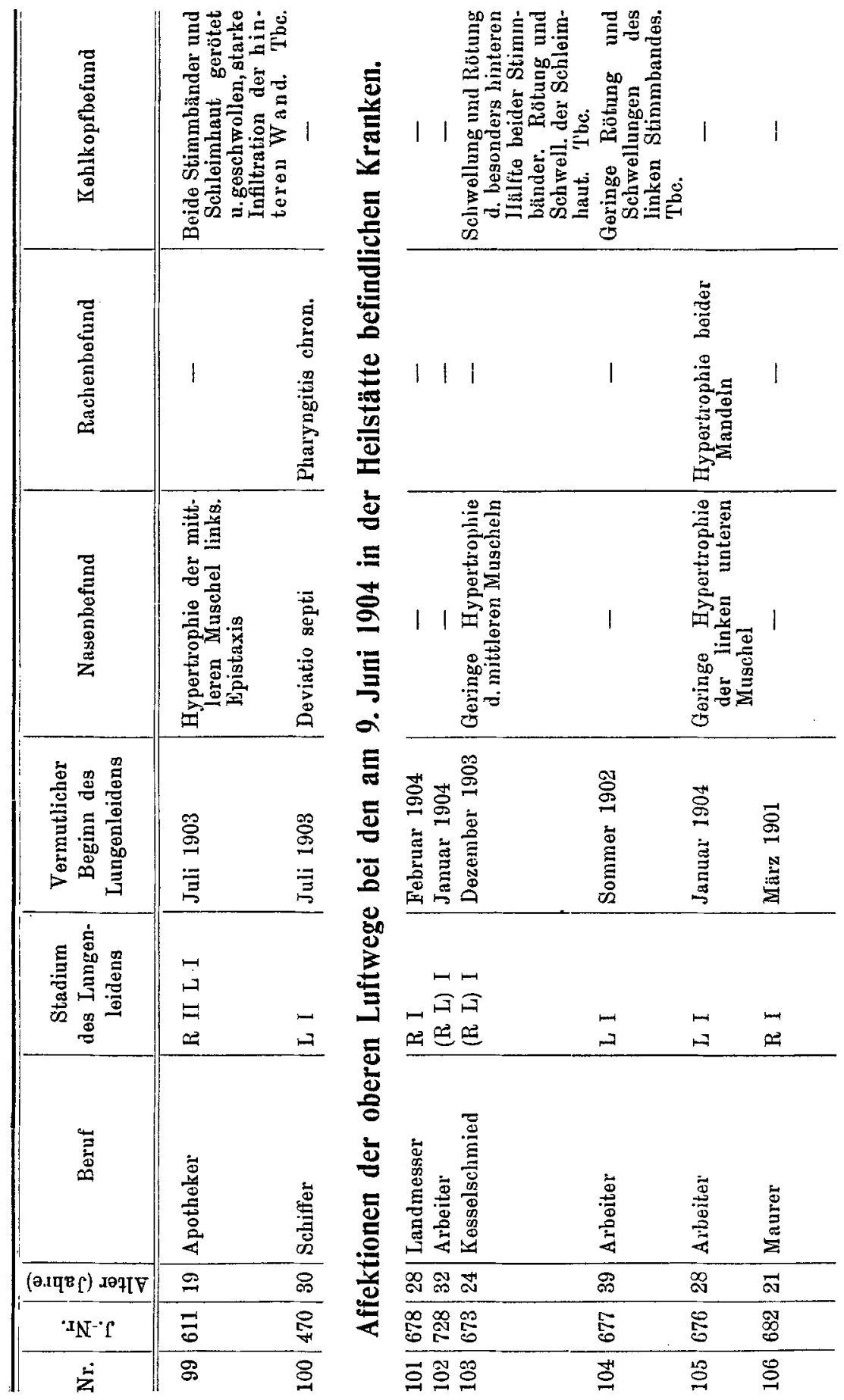


13] Die Affoktionen d. oberen Luftwege bei Phthisikern in den Anfangsstadien. 49
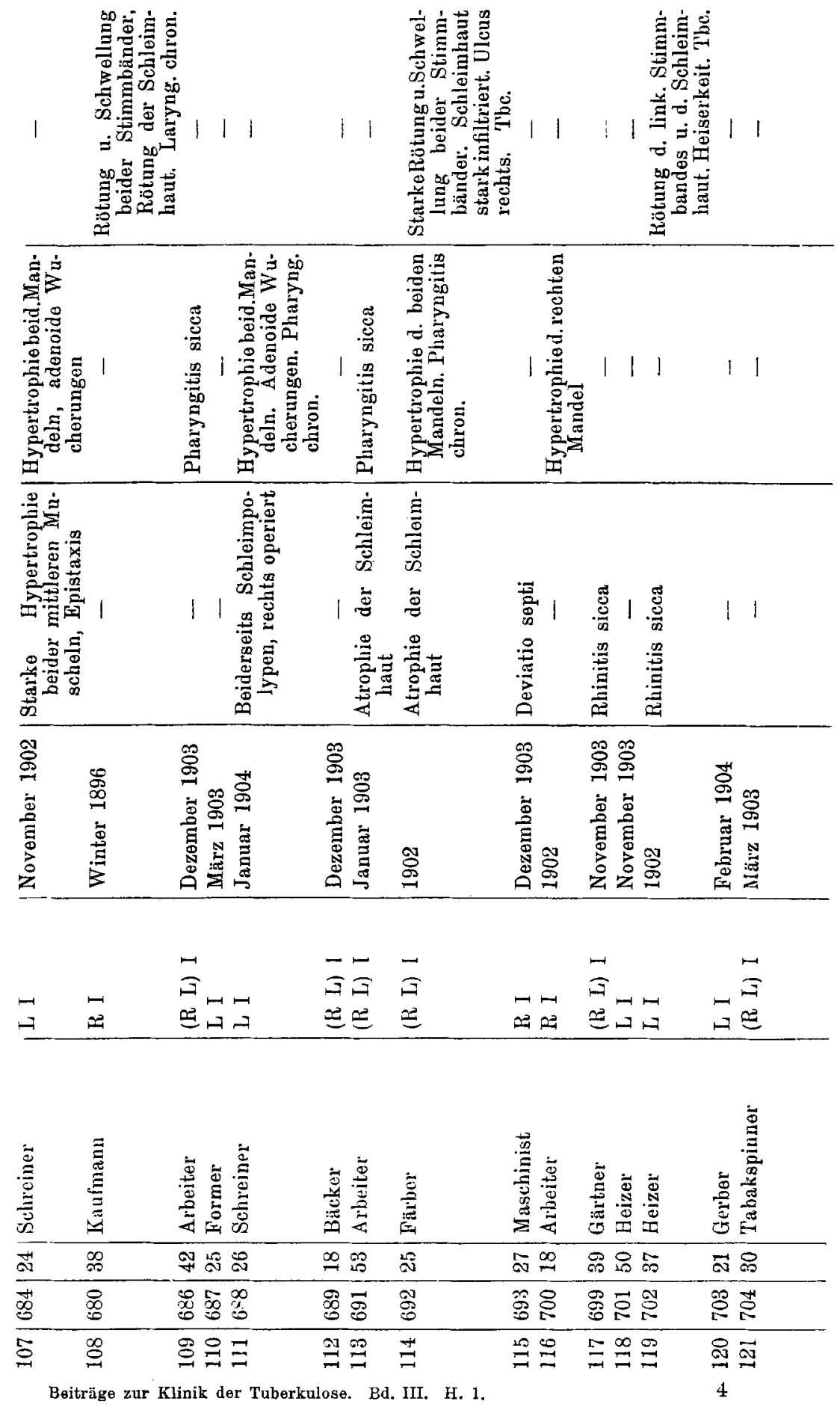


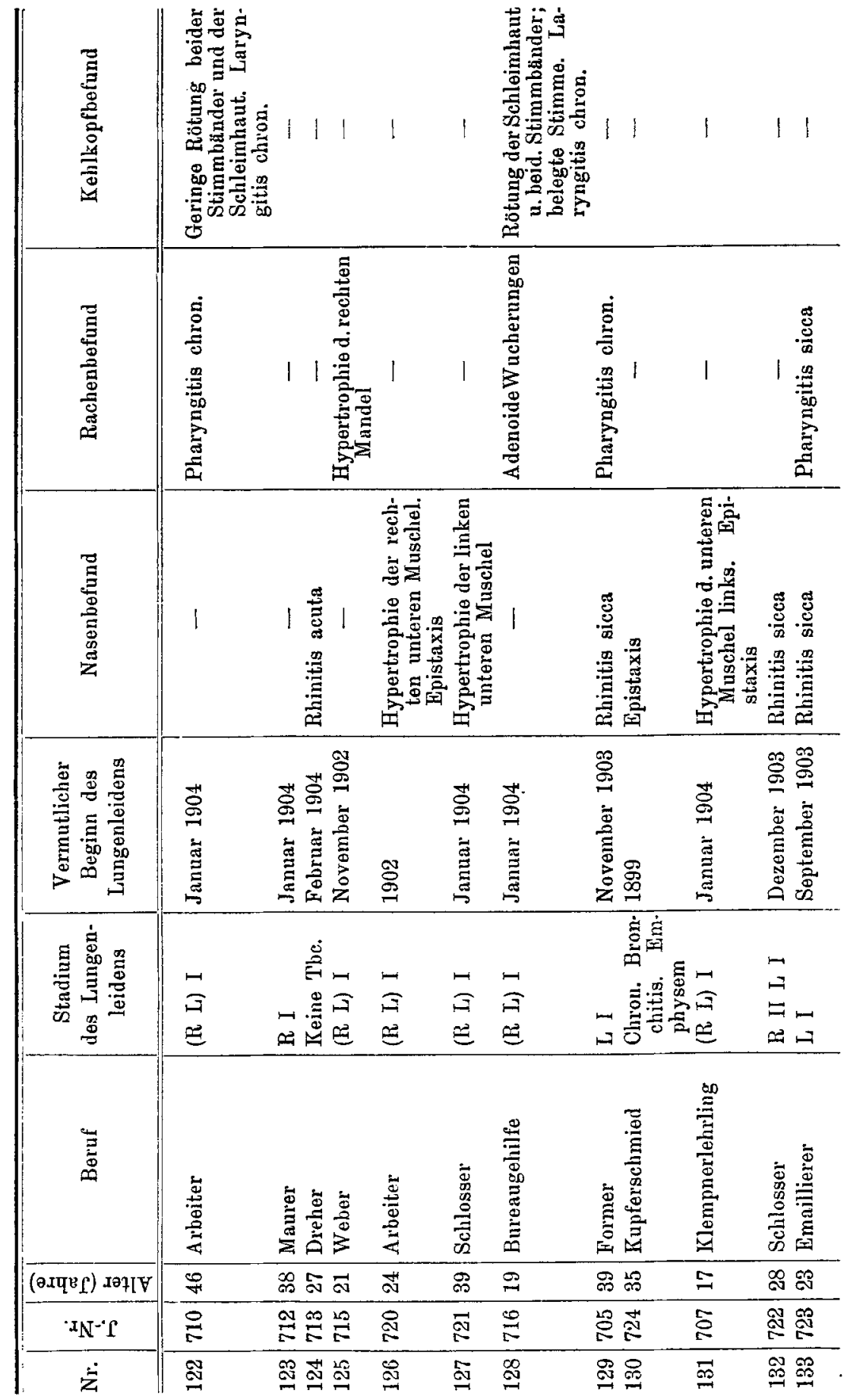


15] Die Affektionen d. oberen Luftwege bei Phthisikern in den Anfangsstadien. 51
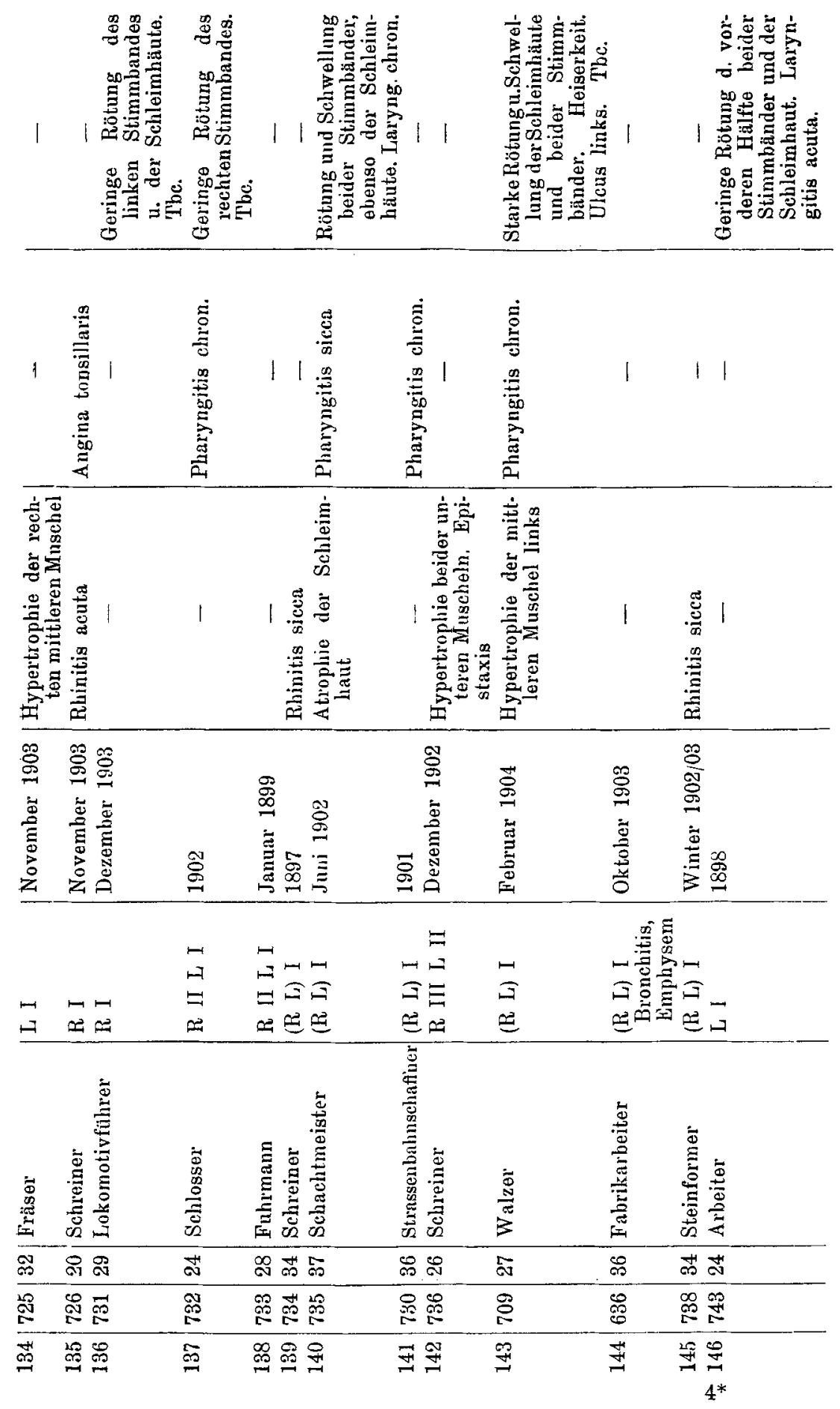


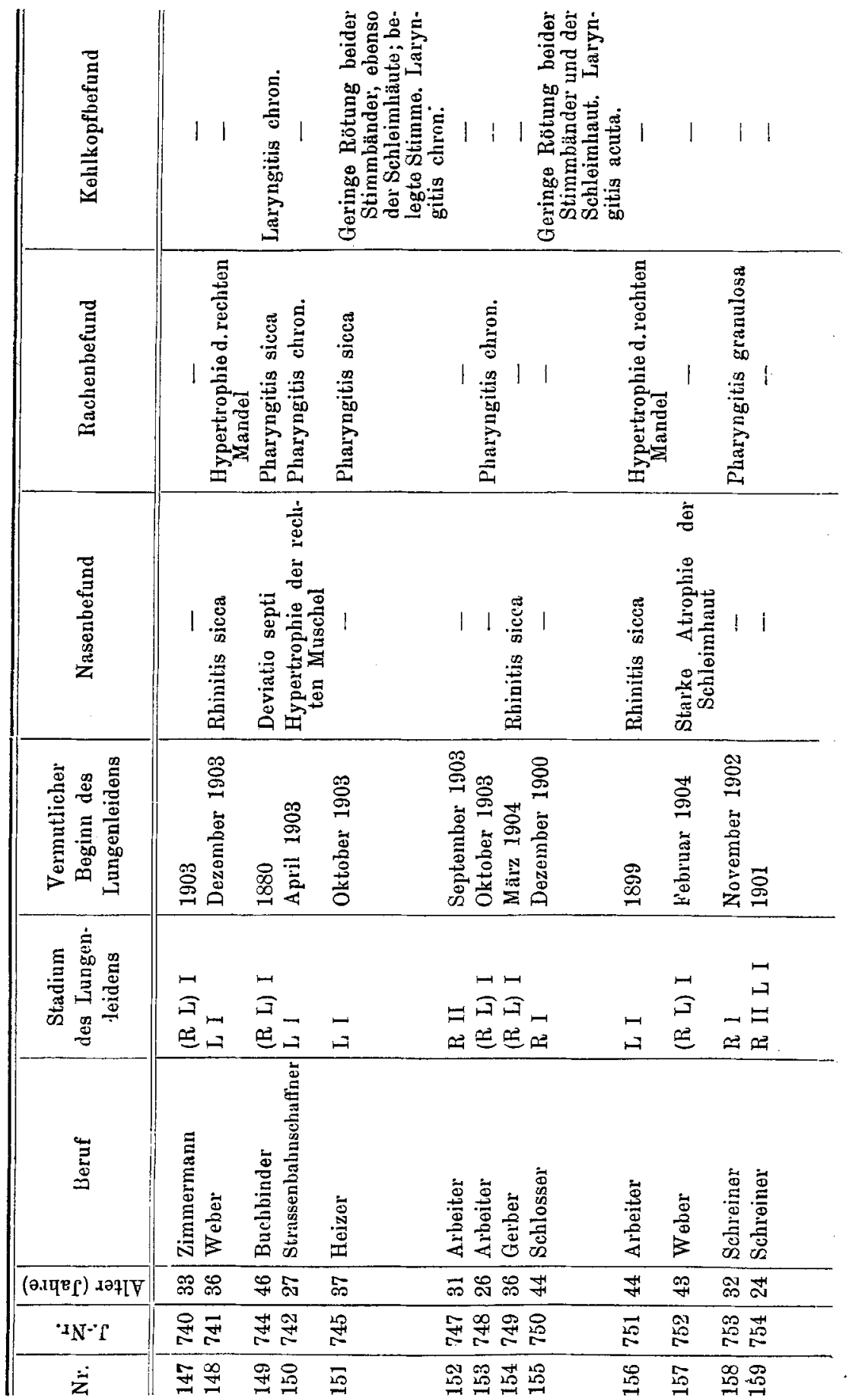


17] Die Affektionen d. oberen Luftwege bei Phthisikern in den Anfangsstadien. 53
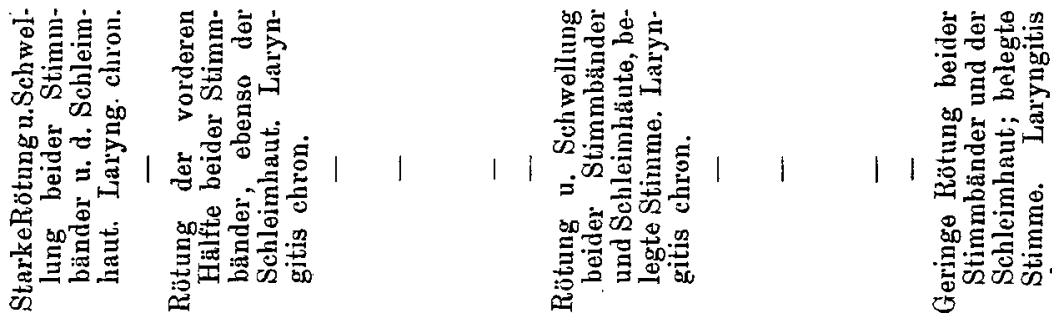

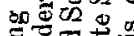

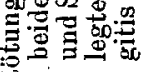

等节

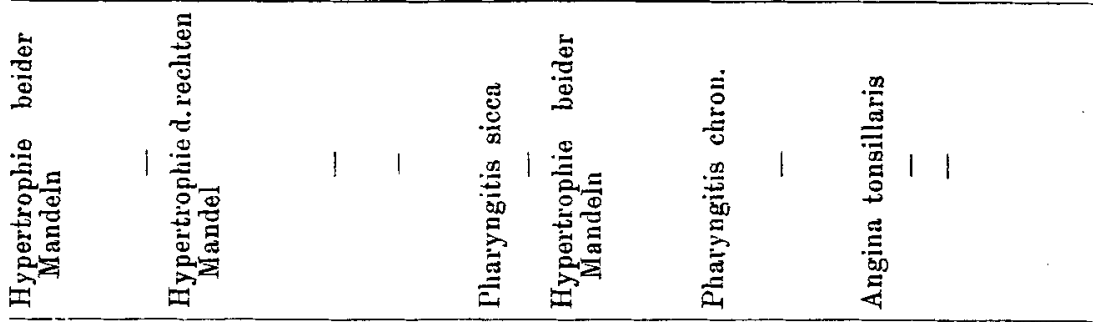

\begin{tabular}{|c|c|c|c|c|c|c|c|}
\hline 1 & 11 & 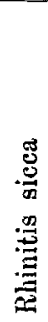 & 1 & 111 & 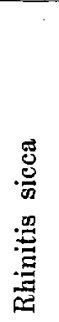 & 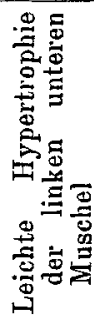 & 111 \\
\hline 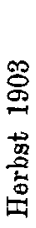 & 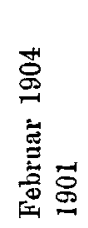 & 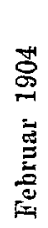 & $\stackrel{5}{\stackrel{5}{\infty}}$ & 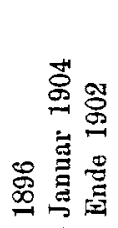 & 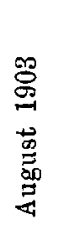 & 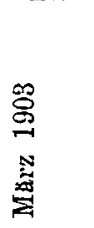 & 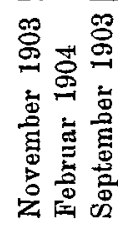 \\
\hline 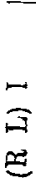 & $\begin{array}{l}\varpi= \\
\Xi \Xi \\
\Leftrightarrow \Xi\end{array}$ & \multicolumn{3}{|c|}{ 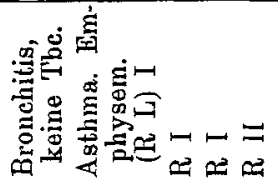 } & \multicolumn{2}{|c|}{ 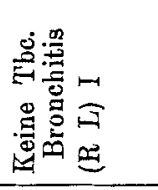 } & 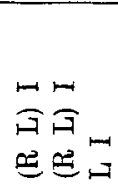 \\
\hline
\end{tabular}

\begin{tabular}{|c|c|c|c|c|c|c|c|}
\hline 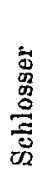 & 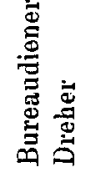 & 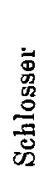 & 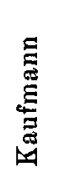 & 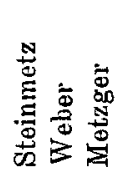 & $\stackrel{5}{\stackrel{5}{\leftrightarrows}}$ & 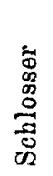 & 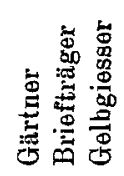 \\
\hline $\mathbb{N}$ & 드용 & 요 & $\overleftarrow{\infty}$ & 品尔 & ६ి & ๙ & 战点 \\
\hline 10 & $\begin{array}{r}5 \\
50 \\
50\end{array}$ & 足 & 8 & $\overrightarrow{0}$ & $\mathscr{8}$ & $\frac{5}{2}$ & 里禺㣽 \\
\hline 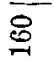 & 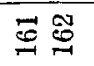 & 范 & Ð & 胥象 & $\mathscr{\varrho}$ & $\stackrel{8}{6}$ & 온존 \\
\hline
\end{tabular}




\begin{tabular}{|c|c|c|c|c|c|c|c|}
\hline 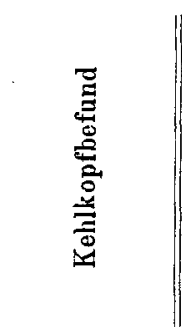 & \multicolumn{3}{|c|}{ 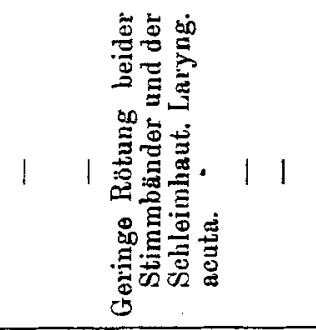 } & \multicolumn{2}{|c|}{ 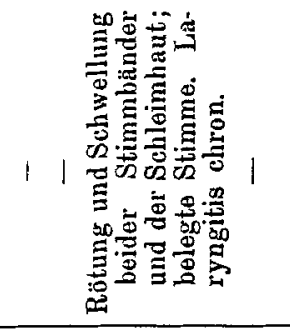 } & \multicolumn{2}{|c|}{ 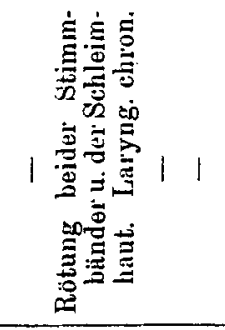 } \\
\hline 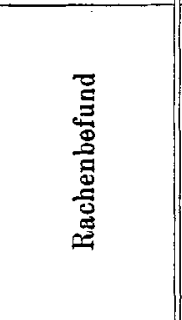 & 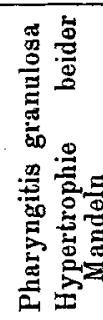 & & 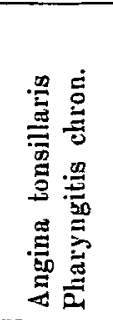 & 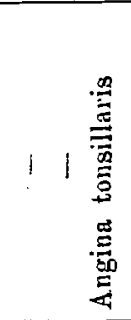 & 1 & 11 & 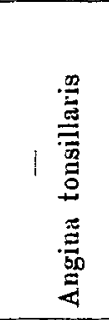 \\
\hline 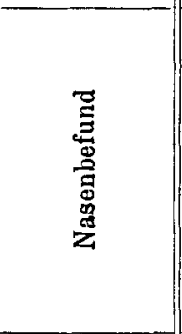 & 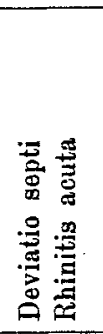 & $i$ & 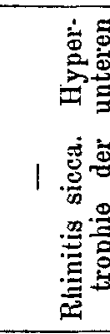 & 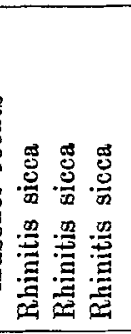 & 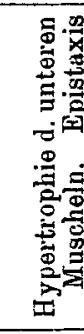 & 11 & 1. \\
\hline 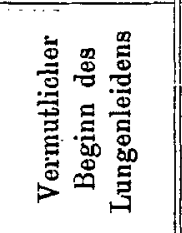 & 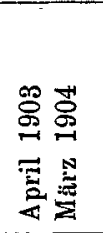 & 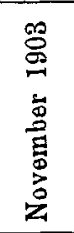 & 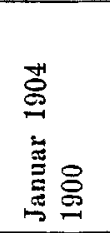 & 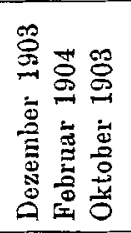 & 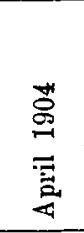 & 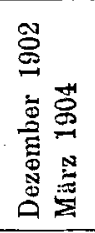 & 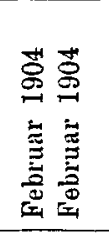 \\
\hline 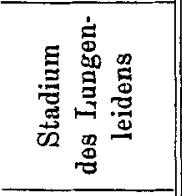 & す心 & 合 & 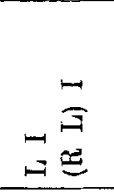 & 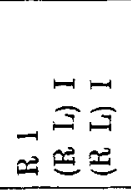 & $\Xi$ & 合 & 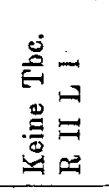 \\
\hline 焉 & 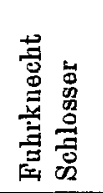 & 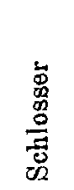 & 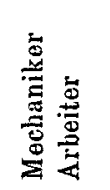 & 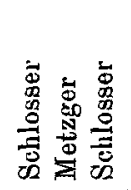 & 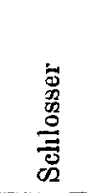 & 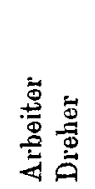 & 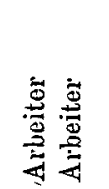 \\
\hline 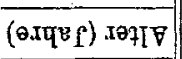 & $\stackrel{\infty}{-\infty}$ & 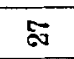 & 9 & 呫果 & $\vec{\nabla}$ & 串 & $5 \infty$ \\
\hline$\cdot \mathrm{x}_{\mathrm{N}} \cdot{ }^{-} \mathrm{C}$ & 로용 & 量 & 哭里 & 呫㣽点 & $\stackrel{\infty}{2}$ & $\stackrel{8}{\circ}$ & $\stackrel{\infty}{\infty}$ \\
\hline$\dot{\vec{z}}$ & 䄈 & 5 & 里5 & 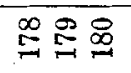 & $\stackrel{\vec{D}}{=}$ & 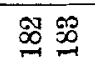 & 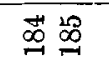 \\
\hline
\end{tabular}


19] Die Affektionen d. oberen Luftwege bei. Phtbisikern in den Anfangsstadien. 55

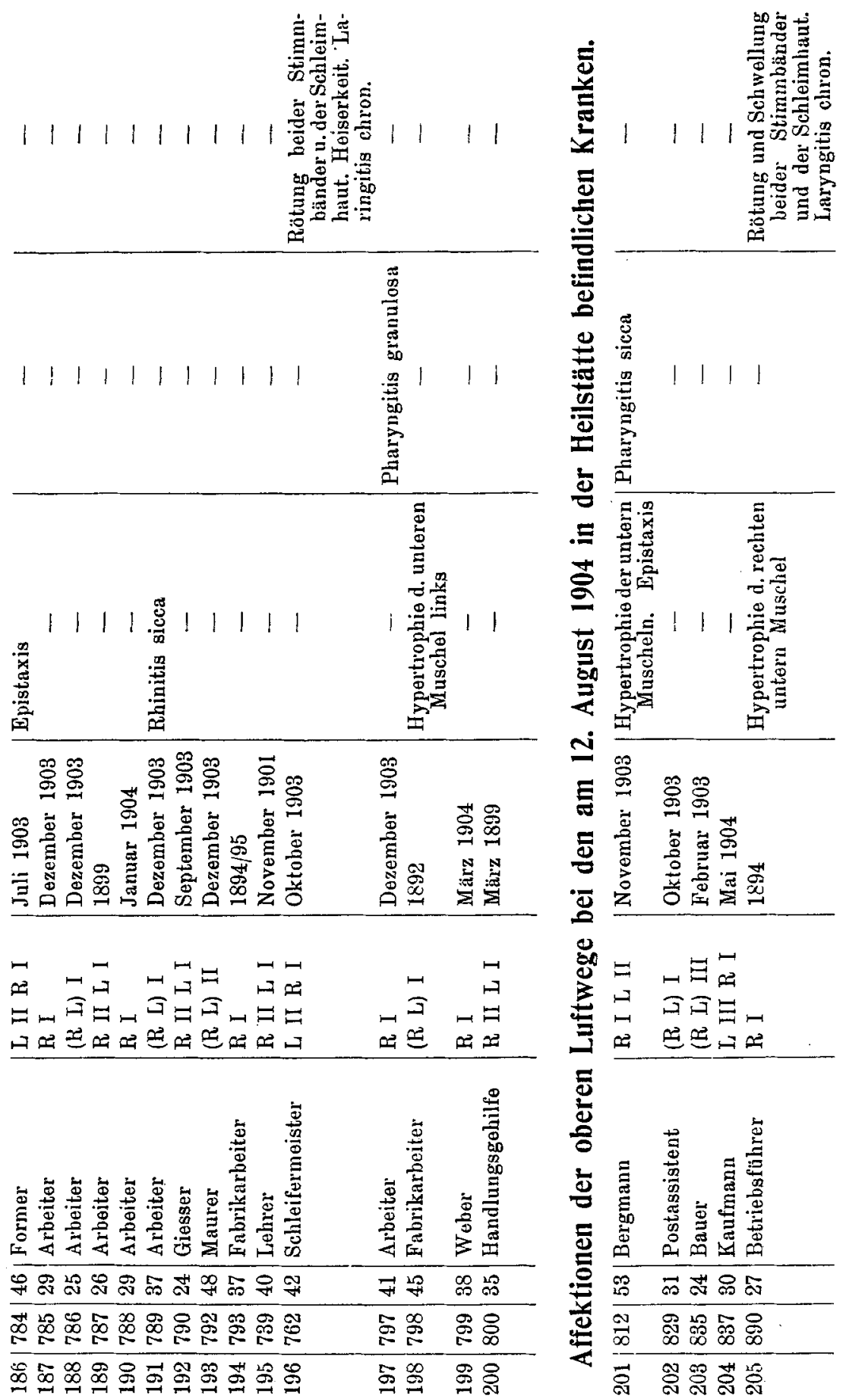




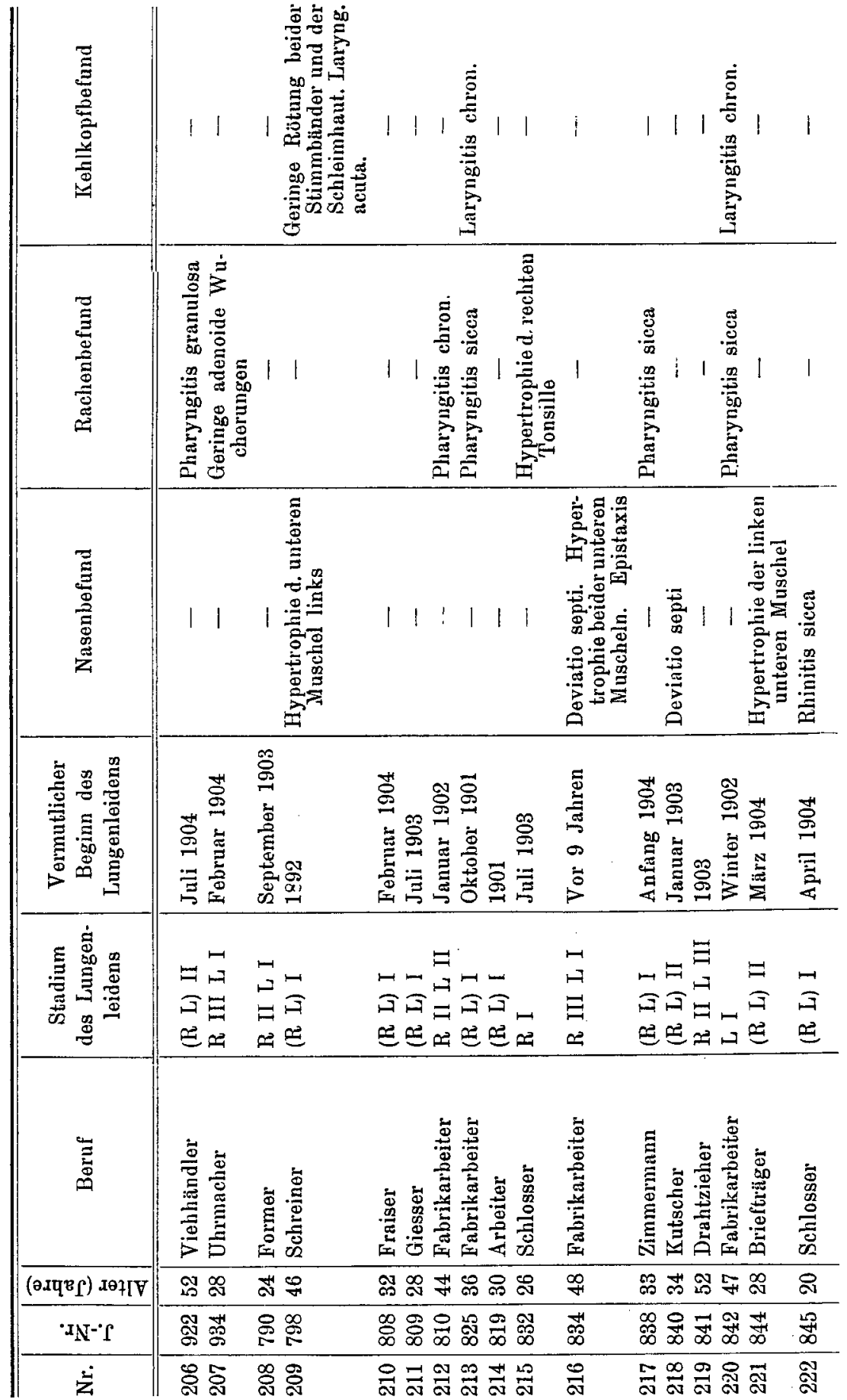


21. Die Affdktionen d. oberen Luftwege bei Phthisikern in den Anfangsstadien. $5 \bar{\tau}$
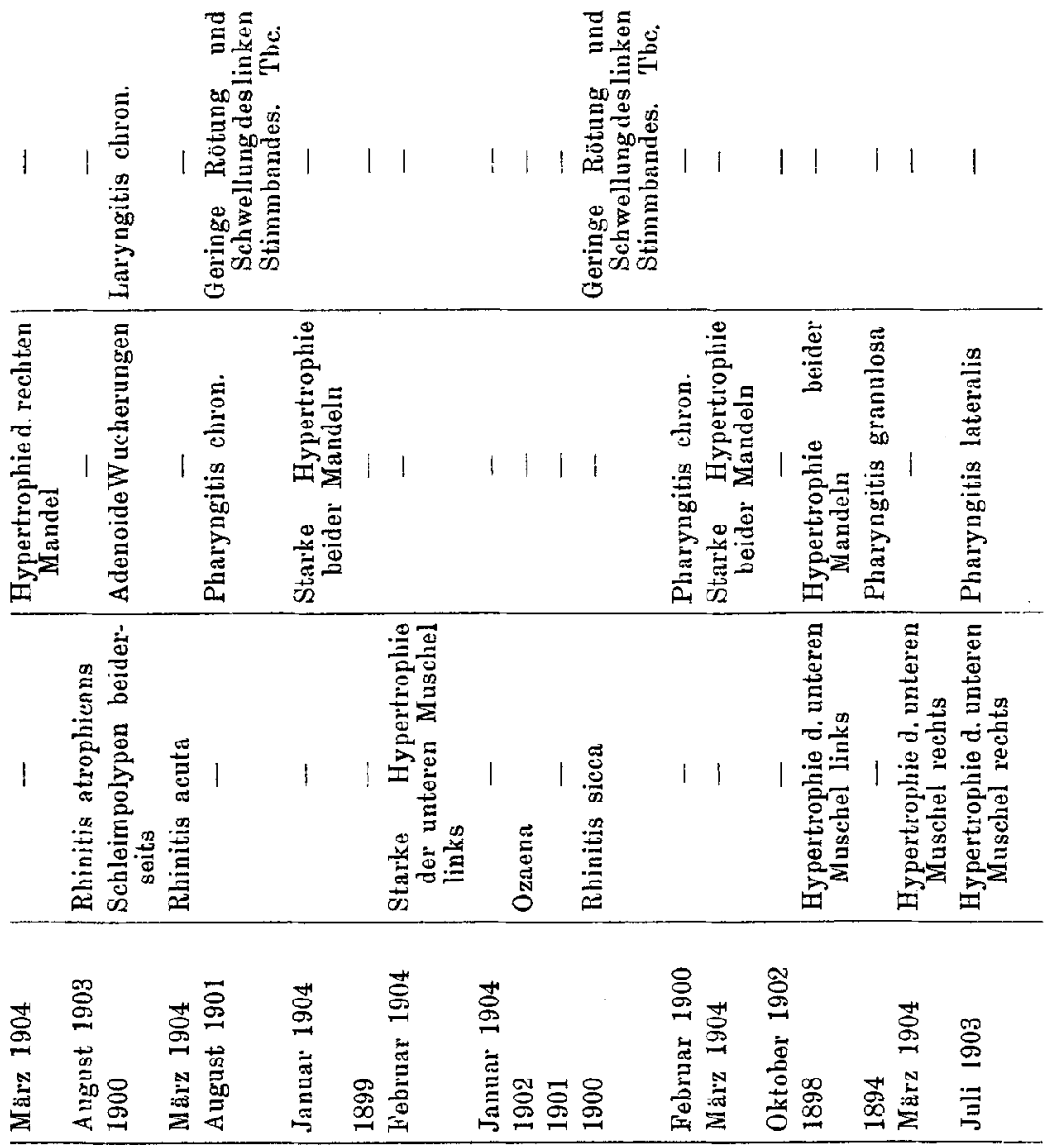

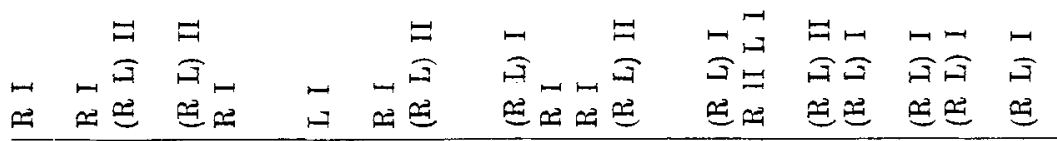

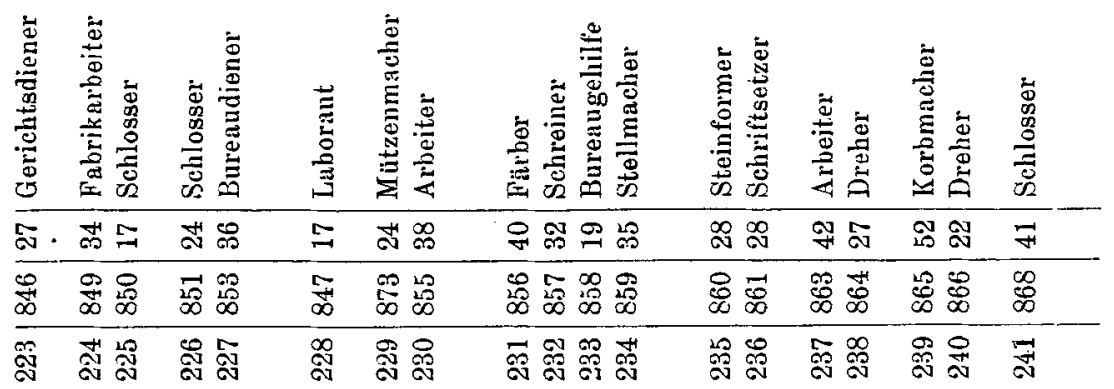




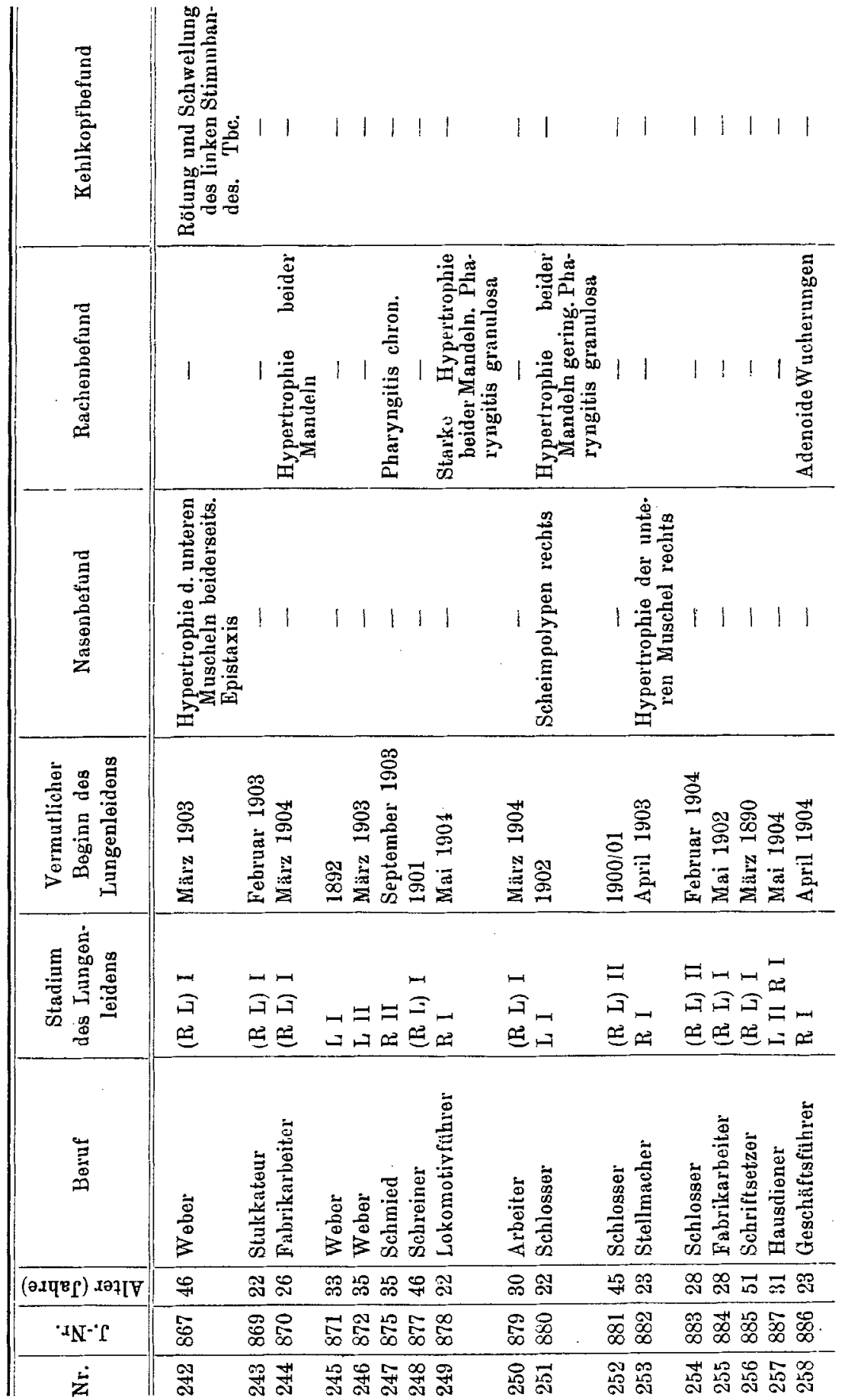


23] Die Affektionen d. oberen Luftwege bei Phthisikern in den Anfangsstadien. 59

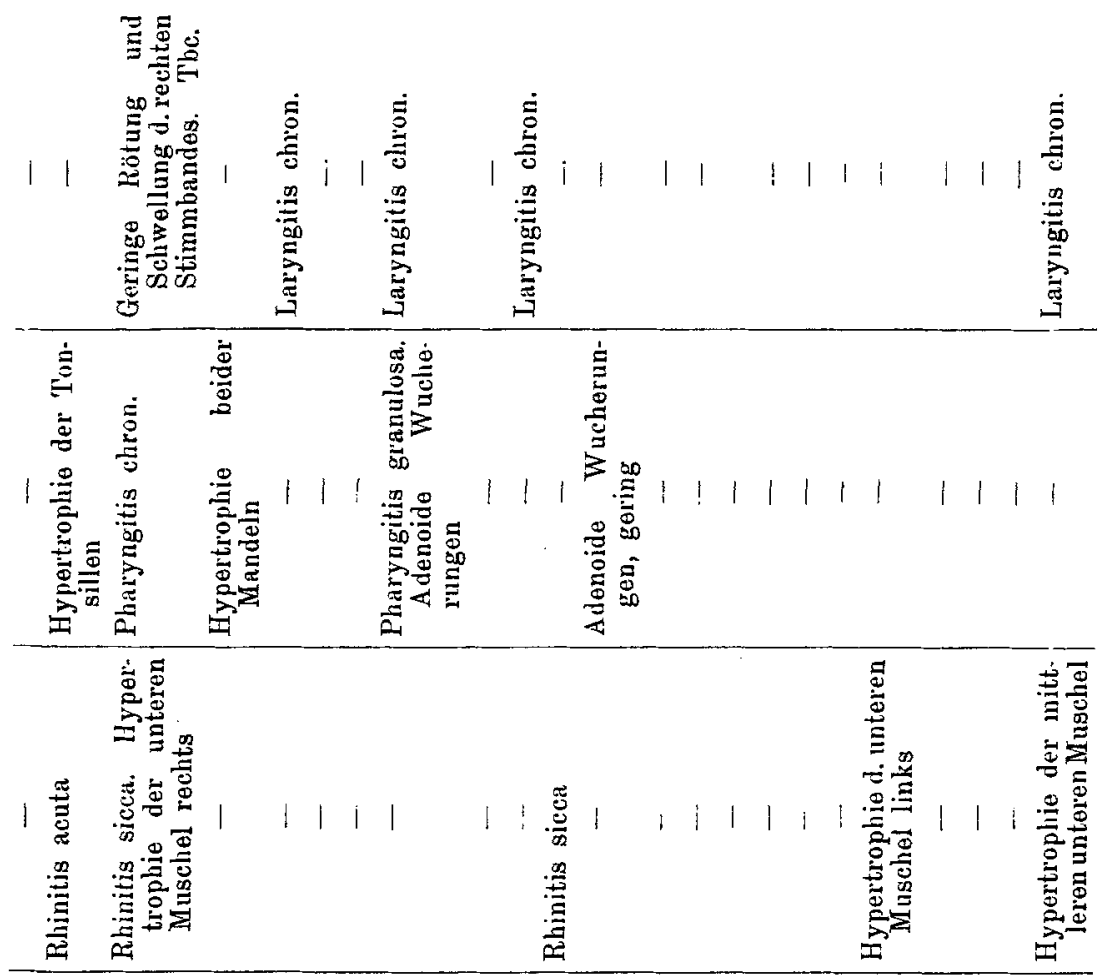

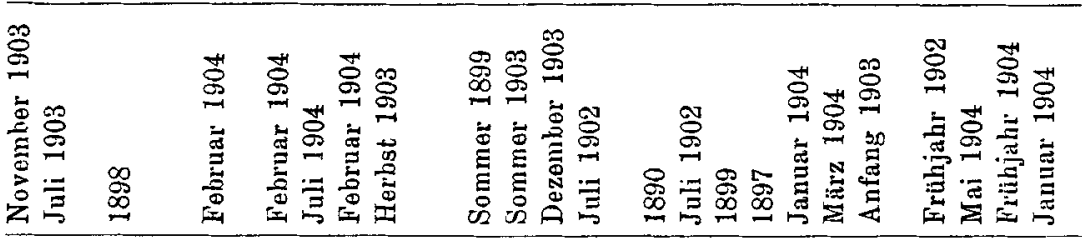

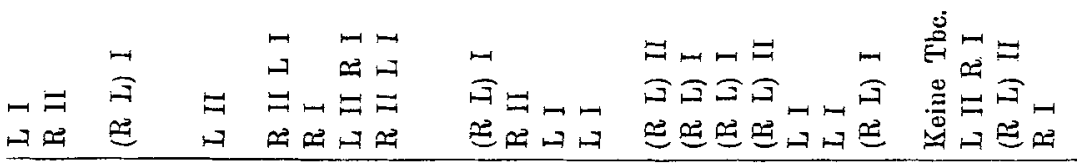

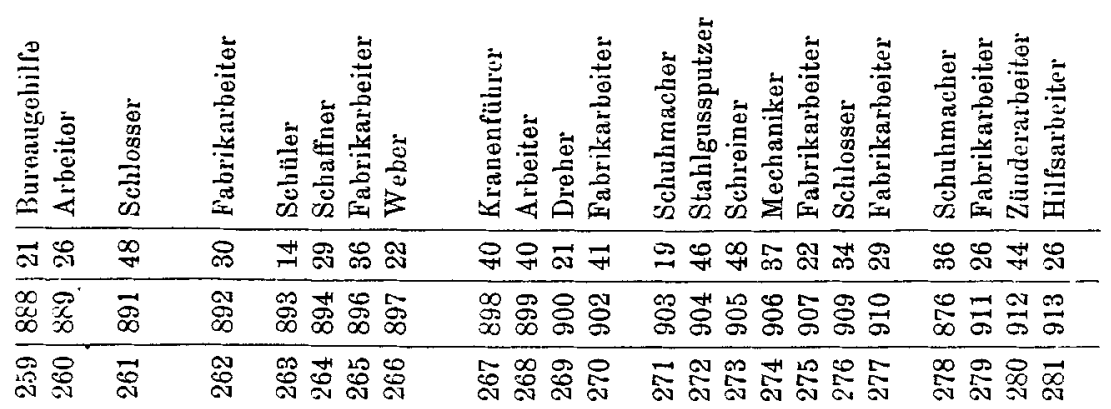




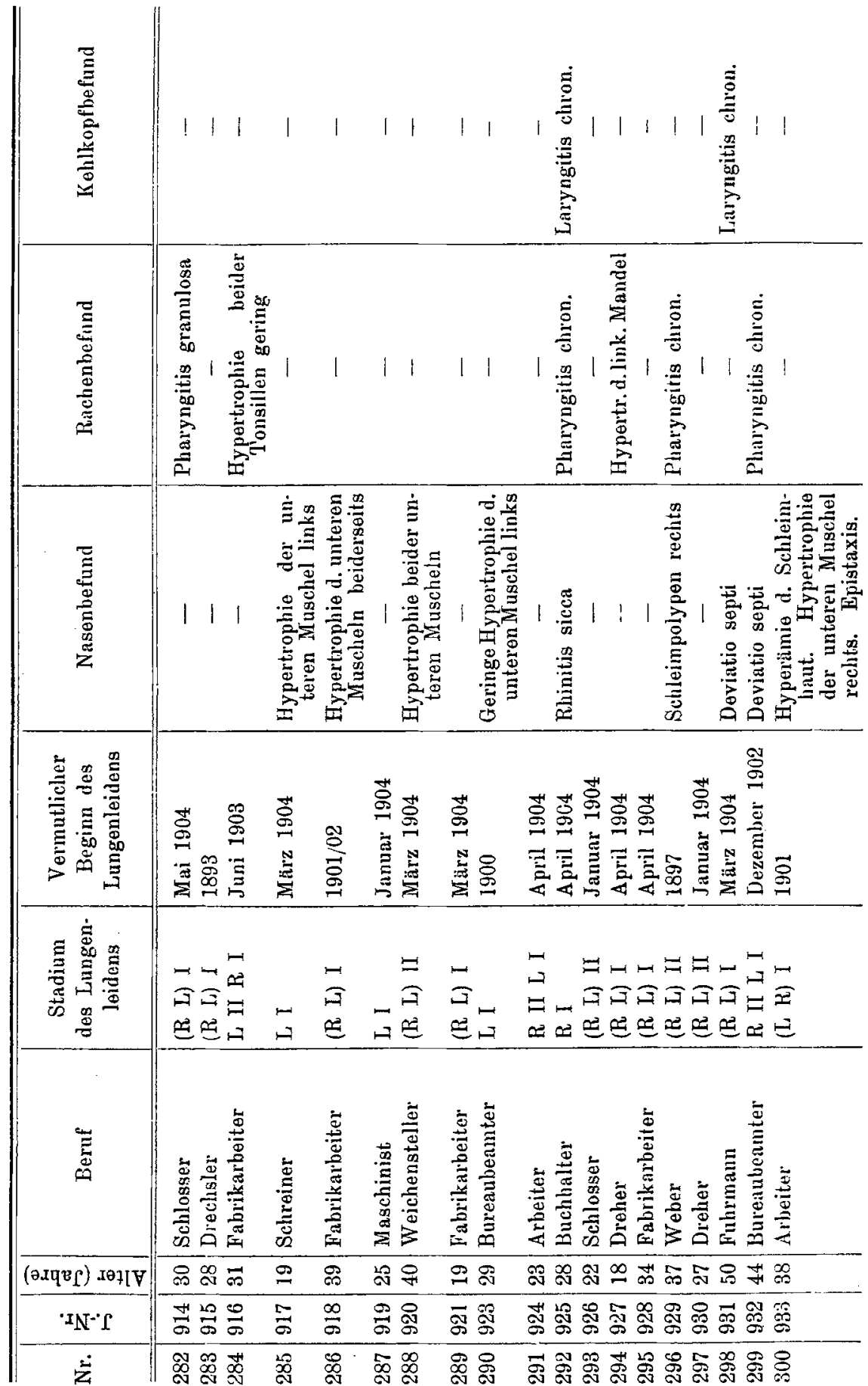


25] Die Affektionen d. oberen Luftwege bei Phthisikern in den Anfangsstadien. 61

Sehen wir uns nun das Ergebnis der Untersuchungen an, und suchen es $z u$ bewerten.

Tabelle A. Zusammenstellung der Untersuehungsbefunde in Zahlen.

\begin{tabular}{|c|c|c|c|c|c|c|c|c|}
\hline \multirow{2}{*}{\multicolumn{3}{|c|}{$\frac{\text { Nase }}{\text { Normaler Befund }}$}} & \multicolumn{3}{|c|}{ Rachen } & \multicolumn{3}{|c|}{ Ke h l k o p f } \\
\hline & & & \multicolumn{3}{|c|}{ Normaler Befund } & \multicolumn{3}{|c|}{ Normaler Befund } \\
\hline & Zahl & $\%$ & & Zahl & $\%$ & & Zahl & $\vdots 0$ \\
\hline Statistik & 31 & & Statistik a & 55 & & Statistik a & 69 & \\
\hline " & 54 & & & 63 & & , & 77 & \\
\hline " & 66 & & & 67 & & & 85 & \\
\hline Surnm: & 151 & ca. $50^{\circ} \%$ & Summa & 185 & $61,7^{\circ}: 0$ & Summa & 231 & $77 \%$ \\
\hline
\end{tabular}

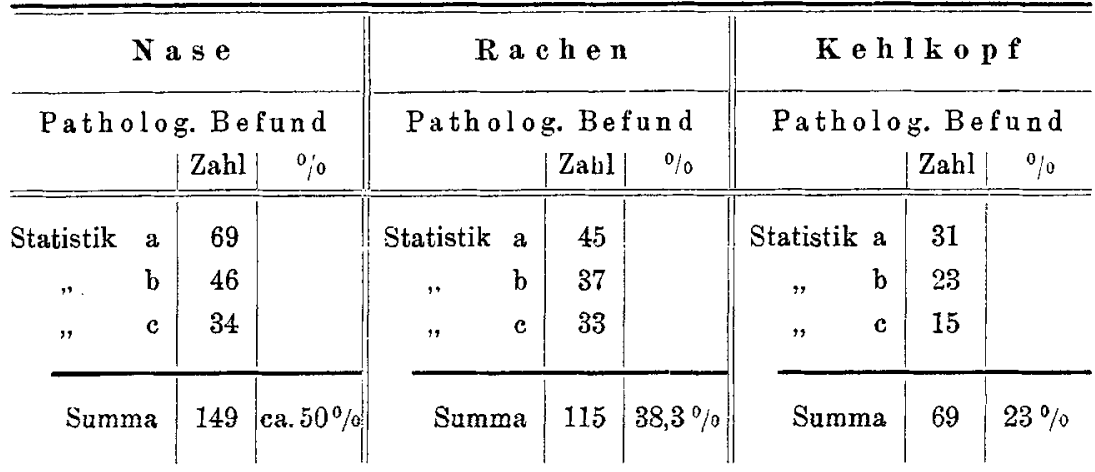

Bei der Betrachtung einer jeden der drei Eintagstatistiken fällt zunächst die bei allen zu konstatierende Abnahme der Erkrankungszahlen proportional der tieferen Lage des befallenen Abschnittes auf. Der oberflächlichste und für die Aussenluft zunächst in Betracht kommende Teil des Atmungstraktus - die Nase - zeigt den höchsten Erkrankungsprozentsatz 50\%; dieser wird anf dem Wege der Inspirationsluft in die Tiefe geringer und beträgt für den Rachen $38,3^{0} \%$, für den Kehlkopf $23 \%$.

Die Erklärung hierfür dürfte wohl in der mehr oder weniger geschützten Lage der einzelnen Teile des Atmungsweges und deren Funktionen gegeben sein, derart, dass sich Lage, Einrichtung und Tätigkeit des jedesmal höher gelegenen durch eine Verstärkung des natürlichen Schutzes vor Erkrankungen bei den tiefer gelegenen dokumentiert. 
Noch eine weitere interessante Tatsache ergibt die Tabelle A. Die Zahl der pathologischen Veränderungen soweit sie jeden einzelnen Abschnitt der oberen Luftwege betreffen, nimmt von der ersten Statistik zur letzten hin merklich ab: Die Untersuchungen der Nase ergaben in der ersten 69 , in der zweiten 46 , in der dritten 34 Affektionen; der Rachen ist in der Aufstellung a) $45 \mathrm{mal}$, in b) $37 \mathrm{mal}$, in c) $33 \mathrm{mal}$ erkrankt; der Kehlkopf zeigt in der ersten Untersuchungsreihe 31 , in der zweiten 23 , in der dritten 15 Affektionen.

Ich glaube den Grund hierfür liefert der Unterschied der Jahreszeit, in welcher die Beobachtungen angestellt wurden. Die Untersuchung der ersten 100 Patienten fand zu Beginn des Monates März statt, zu einer Zeit, wo die Gelegenheit zu Katarrhen reichlich gegeben ist, und die schon bestehenden chronischen durch die rauhe Witterung ungünstig beeinflusst werden. Die folgenden am 9 . Juni erhobenen Befunde zeigen durch die geringere Zahl der krankhaften Veränderungen schon den Einfluss der milderen Frühjahrsluft, während die A ugustresultate bei den diesjährigen vielen, "schönen Tagen" im Juni und Juli die sinkende Erkrankungsziffer noch zunehmen lassen.

Diese Tatsache dürfte wohl unter anderem auch zeigen, dass eine grosse Reihe von Affektionen des Kehlkopfes bei Tuberkulösen nicht als spezifisch anzusehen ist und dass es keinewegs gerechtfertigt ist, jede Halsaffektion eines Phthisikers a uf die Wirkung des Tuberkelbacillus zurückzuführen. Bei einer so chronisch und hartnäckig verlaufenden Affektion, wie es die Larynxtuberkulose ist, würde meines Erachtens sicherlich nicht ein solch merklicher Rückgang der Erkrankungszahl innerhalb so kurzer Zeit selbst bei dem Einflusse besserer Witterungsverhältnisse eintreten, wenn es sich nicht um einen grösseren Prozentsatz katarrhalischer Erkrankungen handelte, die sich nach allen Erfahrungen bei günstigen äusseren Verhältnissen leicht bessern.

Tabelle B. Zusammenstellung der einzelnen Diagnosen in Zahleu.

I. $\mathrm{N}$ a $\mathrm{s}$ e.

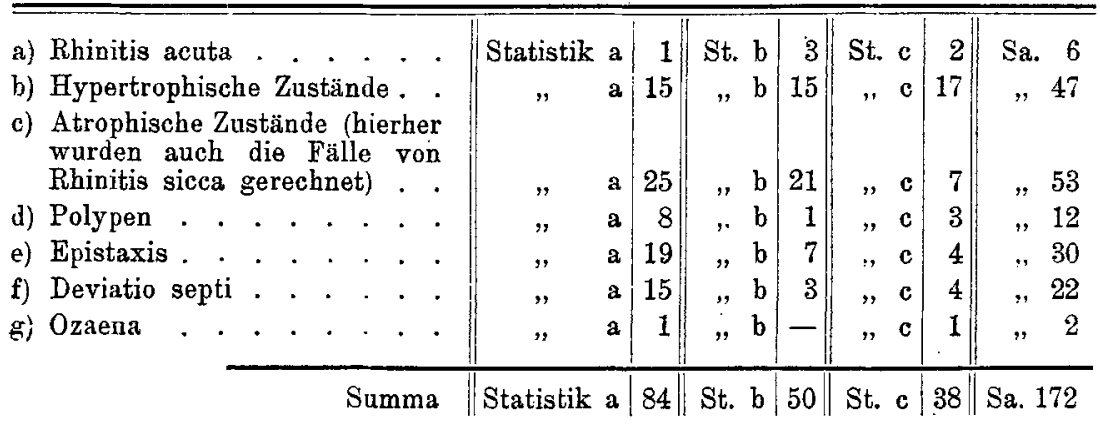




\section{II. $R$ a c h e $n$.}

a) Pharyngitis chron.

(2)

Statistik a 18

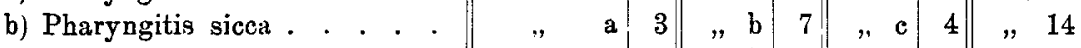

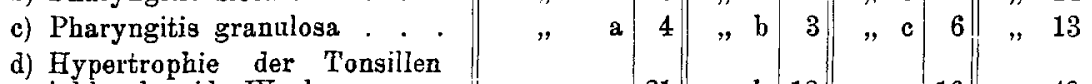
inkl. adenoide Wucherungen .

e) Angina tonsillaris . . . .

f) Pharyngitis lateralis. . .

\begin{tabular}{l||l|l|l|l|l|l|l} 
& & & & & & & \\
\hline Summa & Statistik a & 48 & St. b & 37 & St. c & 36 & Sa. 121
\end{tabular}

III. $K$ e h l k opf.

\begin{tabular}{|c|c|c|c|c|c|c|c|}
\hline 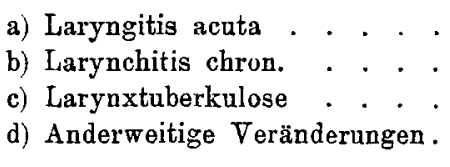 & $\begin{array}{cc}\text { Statistik } & \text { a } \\
, & \mathbf{a} \\
, & \mathbf{a} \\
, \quad & \mathbf{a}\end{array}$ & $\left|\begin{array}{r}2 \\
21 \\
4 \\
6\end{array}\right|$ & $\begin{array}{cc}\text { St. } & \mathrm{b} \\
, & \mathrm{b} \\
, & \mathrm{b} \\
, & \mathrm{b}\end{array}$ & $\begin{array}{r}3 \\
13 \\
7 \\
-\end{array}$ & $\begin{array}{ll}\text { St. } & \mathbf{c} \\
\Rightarrow \mathrm{c} \\
\Rightarrow \mathrm{c} \\
\Rightarrow \mathrm{c}\end{array}$ & $\begin{array}{r}1 \\
10 \\
4 \\
-\end{array}$ & $\begin{array}{lr}\text { Sa. } & 6 \\
, & 44 \\
, & 15 \\
, & 6\end{array}$ \\
\hline & Statistik a & 33 & St. $b$ & 23 & St. c & 15 & \\
\hline
\end{tabular}

Die Tabelle B zeigt die Anzahl der einzelnen Affektionen,

I. Unter den Nasenerkrankungen waren die atrophischen Zustände, unter die auch die Rhinitis sicca aufgezählt wurde, am häufigsten. Hierfür dürfte wohl der Beruf der meisten Patienten verantwortlich gemacht werden. Die grosse Mehrzahl derselben ist als Fabrikarbeiter in Räumen beschäftigt, die nach Angabe der Kranken meist viel inbezug auf Ventilation und Sauberkeit zu wünschen übrig lassen. Wie sehr die Gesundheit der Nase durch eine zu trockene und staubige Luft gefährdet ist, hat neuerlich Freudenthal an der Hand eines grösseren Krankenmaterials gezeigt; er hat unter 110 Fällen $43 \mathrm{mal}$, bei weiteren $108,40 \mathrm{mal}$ eine trockene oder atrophische Rhinitis gefunden. $\mathrm{Er}$ ist geneigt mit Rücksicht auf die grosse Zahl der Affektionen, dieselben als ein kansales Moment für die Lungentuberkulose aufzufassen.

Was die Angabe von subjektiven Beschwerden bei unsern Patienten anlangt, so möchte ich die grosse Verschiedenheit derselben betonen. Ich habe mich sehr oft gewundert, wie Kranke trotz starker hypertrophischer Zustände auf Befragen erklärten, dass sie gut durch die Nase atmen könnten, während andere mit sehr weiten atrophischen Nasen ïber das Gegenteil klagten. Ich finde diese Beobachtung von Freudenthal bestätigt. Dieser sieht für die erstere Erscheinung 
den Grund darin, dass "die Hypertrophien leichter dem Luftstrom nachgeben und so die physiologische Tätigkeit der Nase ermöglichen “, während er den "Lufthunger" bei atrophischer Nase der Unfähigkeit zuschreibt, die Inspirationsluft zu "assimilieren“.

II. Die Erkrankungen des Rachens stehen begreiflicherweise in engem Zusammenhange mit den pathologischen Zuständen der Nase; ist diese in ibrer physiologischen Tätigkeit geschädigt, so wird in einer grossen Anzahl der Rachen mitaffiziert. Unter den von uns untersuchten Patienten litt ein beträchtlicher Teil an chronischem Katarrh. Bei den meisten ist neben übermässigen Tabakgenuss der leider unter unserer arbeitenden Bevölkerung weit verbreitete Alkoholmissbrauch, der selbst, wie die Berichte der Heilstättenärzte immer klagen, bei der Anstaltsbehandlung nur mit Mühe bekämpft wird, als die Ursache der Frkrankung zu betrachten.

Bemerkenswert ist der hohe Prozentsatz der Tonsillenhypertrophien (die begleitenden Pharyngitiden sind nicht besonders aufgezeichnet) den unsere Statistik dartut. Dies ist um so wichtiger als neuerdings von mehreren Seiten die Mandel als Eingangspforte für die Tuberkelbacillen bezeichnet wird.

So schreibt Beckmann (das Eindringen der Tuberkulose und ihre rationelle Bekämpfung. Berlin 1904 S. Karger). „Alle diese Beobachtungen und Tatsachen zwingen uns die Anschauung auf, dass mit dem Heranwachsen des Individuums die Antikörper gegen die Tuberkulose sich mehren, und dass im späteren Leben die Tuberkulose dem Menschen hauptsächlich dadurch gefährlich wird, dass akute Rachenmandelentzündungen alte in Halsdrüsen deponierte Tuberkelherde frei machen und der Lungenspitze zuführen, oder dass dort abgekapselte Herde durch die frische pyogene Infektion wieder aufleben."

E. A u frecht (Über die Lungenschwindsucht, Magdeburg 1904 Verlag Faber) vertritt auf Grund von mitgeteilten anatomischen Untersuchungen bei Sektionen und an exstirpierten Tonsillen, sowie gestützt auf Tierversuche, einen ähnlichen Standpunkt: „Die hauptsächlichste Eintrittspforte des Tuberkelbacillus in den menschlichen Körper sind die Mandeln, und die Zeit des Eintrittes fällt sehr häufig in ảie früheste Kindheit; von den Mandeln dringt der Bacillus in die Hals- und weiter in die Mediastinaldrüsen". Er will in jedem Falle die "geschwollenen Mandeln“ entfernt wissen.

Ich erinnere ferner an die schönen Versuche Grobers (Deutsch. Archiv. für klin. Ned. Bd. LXVIII), welcher in die Tonsillen von Hunden chinesische Tusche injizierte und dieselbe auf dem Lymph- 
29] Die Affektionen d. oberen Luftwege bei Phthisikern in den Anfangsstadien. 65

wege bis zur Pleura verfolgen konnte, gleichzeitig aber auf die tuberkulöse Infektion der Lunge von den Tonsillen und Halsdrüsen aus hinwies, bei welcher der Bacillus gewöhnlich den Weg über die Pleurakuppen nimmt und der mehr behinderten und länger dauernden Bahn zu den Bronchialdrüsen und von hier zu den Pulmones vorzieht.

Nach Untersuchungen von $\mathrm{Kr}$ ü $\mathrm{ckmann}$ (Über die Beziehungen der Halsdrüsentuberkulose zu den Tonsillen. Virch. Arch. Bd. 138), Friedmann (Über die Bedeutung der Gaumentonsillen von jungen Kindern als Eingangspforte für die tuberkulöse Infektion. Deutsche med. Wochenschr. 1900 Nr. 24), Strassmann (Über die Tuberkulose der Tonsillen. Virch. Arch. Bd. 138), Scheibner (Bilden die Tonsillen häufige Eingangspforten für die Tuberkelbacillen? Zi eglers Beiträge $1899 \mathrm{Bd}$. 26), Aufrecht (Über die Lungenschwindsucht Magdeburg 1904) darf es wohl als feststehend angesehen werden, dass eine Infektion der erwähnten Rachenteile in der Tat vorkommt, und in gleicher Weise auf dem experimentell von Gerber gezeigten Wege absteigend sich Bahn bricht.

Or th (Experimentelle Untersuchungen über Fütterungstuberkulose. Virch. Arch. Bd. 76) hat bei seinen Fütterungsversuchen unter neun Fällen einmal, Baumgarten aber in $100 \%$ primäre Tonsillartuberkulose beobachtet; Ito (Berl. klin. Wochenschr. Nr. 32) hält auf Grund seiner pathologisch-anatomischen Untersuchungen die tuberkulöse Affektion der Mandeln zwar für selten; erblickt aber den Grund hierfür darin, dass die Tonsillen für die Tuberkelbacillen einmal leicht durchgängig sind und ferner, dass der gesetzte tuberkulöse Herd frühzeitig abheilt.

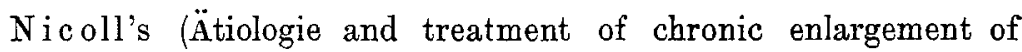
lymphatic glands, with special reference to those of the neck. Glasgow medical journal) Untersuchungen von 500 geschwollenen Halsdrïsen bei Kindern ergab in $80 \%$ die Anwesenheit virulenter Bacillen und Dmochowsky (Zieglers Beiträge Bd. 10 und 16) sah in den zu den Halsdrüsen führenden Lymphgefässen die Tuberkelbacillen frei im Lymphstrom auf der Wanderung.

Die Durchsicht der Literatur ergibt eine grosse Zahl von Fällen bei welchen der Tuberkelpilz den erwähnten Weg vom lymphatischen Rachenringe unter Vermittelung der Lymphdrüsen am Halse zur Lunge eingeschlagen hat. Bemerkenswert hierfür ist auch die Beobachtung, dass Patienten, welchen die tuberkulösen Halsdrüsen exstirpiert waren, später in einem beträchtlichen Prozentsatze phthisisch erkrankten und zwar nach dem Zitate von Grober bei

Beitrüge zur Klinik der Tuberkulose. Bd. III. H. 1 . 


$\begin{array}{lr}\text { Riedel-Göttingen } & \text { 1. Mitteilung in } 15 \% \\ \text { Schnell-Bonn } & \text { 2. Mitteilung in } 12 \% \\ \text { Fränkel-Wien } & \text { in } 12 \% \\ \text { Krisch-Breslau } & \text { in } 10 \% \\ \text { v. Noorden-Tübingen } & \text { in } 14 \% \\ & \text { in } 25,3 \%\end{array}$

das heisst also, dass $14,3 \%$ der wegen tuberkulöser Halsdrüsen Operierten später von Lungenphthise befallen warden; hier dürfte wohl eine descendierende Tuberkulose das Nächstliegendste sein.

Auch noch andere Teile von Rachen und Mund werden in der letzten Zeit als Eingangspforten für die Infektion angesehen und bieten weiteren Grund dafür, dass wir den obersten Luftwegen unsere Aufmerksamkeit in erhöhtem Masse schenken. Westenhöffer (Berl. klin. Wochenschr. $1904 \mathrm{Nr} .7$ u. 8) glaubt, dass bei Kindern wäbrend der Dentition die Mikroorganismen ihren Weg durch die wunde Schleimhaut nehmen; Heller hat die Zungenbalgdrüsen als solche nachgewiesen; $\mathrm{F}$ ischer (Vortrag im Verein niederrheinischwestfälischer Kinderärzte: Autoreferat Münch. med. Wochenschr. 1904 Nr. 34) sezierte einen Mann mit geschüriger Tuberkulose der Zungenwurzel und Tonsillen, der gleichzeitig an tuberkulöser Meningitis und Kniegelenkserkrankung litt.

Auch die von Volland seit Jahren vertretene Ansicht, dass der Tuberkelbacillus sich durch die Haut Eingang in den Körper verschaffe, muss hier erwähnt werden, da ihm als Prädilektionsstelle für die Infektion die besonders bei Kindern so häufigen wunden Nasen und Lippen gelten.

Wenn auch den mitgeteilten Ansichten über den Beginn der tuberkulösen Erkrankung das gegenteilige Urteil anderer verdienter Forscher gegenübersteht, so kann doch die Möglichkeit der erwähnten Art der Infektion vor allem die vom lymphatischen Rachenringe aus nach den Tierversuchen Grobers nicht von der Hand gewiesen werden. Sie hat im Gegenteile vieles für sich und vermag manche vielumstrittene Frage der Phthisiogenese der Lungen - ich erwähne nur die häufigste anfängliche Lokalisation in den Spitzen - zu erklären.

Ein abgeschlossenes Urteil in dieser Frage wird man sich heute noch nicht erlauben dürfen; ist aber die Menzersche Theorie vom Entstehen des akuten Gelenkrheumatismus infolge einer Streptokokkeninfektion auf dem Wege durch die Tonsillen, eine Ansicht, die viele Anhänger besitzt, richtig, warum sollte dann der Tuberkelbacillus nicht in gleicher Weise hier ein bequemes "Entrée" finden. 
III. Ich komme zum III. Teile der Tabelle B.

Die Mehrzahl der erkrankten Kehlköpfe bei unseren Phthisikern imponierte durch katarrhalische Veränderungen und wurde mit der Diagnose Laryngitis chronica belegt. Wie ich schon bei der Erläuterung der Tabelle A bemerkte, glaube ich in der in einer verhältnismässig kurzen Zeit bewirkten guten Beeinflussung unserer Laryngitiden durch die günstigeren Witterungsverhältnisse in den dem Sommer sich nähernden Monaten resp. während dieses selber, einen Grund sehen zu dürfen, die Häufigkeit eines spezifisch tuberkulösen Charakters der Katarrhe auszuschliessen. Gewiss kann durch dieselben Verbältnisse auch eine tuberkulöse Entzündung gebessert werden, bei der Hartnäckigkeit derselben aber sicherlich nicht in so vielen Fällen und innerhalb der erwähnten Zeit.

Die tuberkulöse Erkrankung des Kehlkopfes wurde in 15 Fällen gefunden. Die Häufigkeit ihres Vorkommens in den einzelnen der drei Untersuchungsreihen lässt einen Rückschluss auf event. Rückgang derselben im Sommer nicht zu, eine Bemerkung, welche ich mir mit Rücksicht auf meine Ausführungen beim Kehlkopfkatarrh erlaube. Einen Prozentsatz zu berechnen, ist bei unserem einseitigen Krankenmateriale wertlos. Von den spezifisch-affizierten 15 Patienten standen 13 im Alter zwischen 20 und 40 Jahren, einer war 46 , ein anderer $48 \mathrm{Jahre}$ alt; wir befinden uns hier in Übereinstimmung mit den anderen Autoren. Über das Auftreten der Larynxaffektionen auf der Seite der erkrankten Lunge wie es von Türck, Schrötter, Friedreich, Schech, Schnitzler, Schäffer, Hagen, Kohn und Pfeiffer betont wurde, geben unsere Beobachtungen keinen Aufschluss.

Es wird wahrscheinlich der Einwand erhoben werden, dass unter den als chronisch-katarrhalisch bezeichneten Affektionen vielleicht ein grosser Teil als tuberkulös zu betrachten sei. Wie ich schon andeutete, ist nun hier nicht mit Gewissheit zu entscheiden. $\mathrm{Zu}$ der für die Differentialdiagnose von M. Schmidt empfohlenen Tuberkulininjektion habe ich wenig Vertrauen. Bei unseren Kranken erhielten wir gewiss stets eine Reaktion. Ich glaube aber, dass sie schlechthin in dem erwünschten Sinne nicht zu verwerten ist. Auch F. Blumenfeld (Handbuch der Therapie der chronischen Lungenschwindsucht, Leipzig 1904, J. A. B arth) vertritt diese Ansicht und teilt einen mit Erfolg operierten Fall von Pharynxkarziom an der Hinterwand des Larynx mit, welches auf Grund der positiven lokalen Tb-Reaktion als Larynxtuberkulose diagnostiziert worden war. Die Tuberkulinreaktion ist von so vielen Zufällen und Eiventualitäten ababhängig und begleitet, dass man bei ihrer Verwertung nicht vor- 
sichtig genug sein kann, wie auch eine Reihe von uns später mitzuteilenden Beobachtungen zeigen wird.

Nebenbei mag mir die persönliche Mitteilung gestattet sein, dass. ich mich jedesmal nach den hier mitgeteilten Kehlkopfuntersuchungen. von je 100 Phthisikern an einem Tage mehrere Stunden lang ziemlich unwohl fühlte; ich hatte mehr oder weniger starke Kopf- und Gliederschmerzen, die ich sonst nie gespürt, und war sehr abgespannt. Einmal gelang mir der Nachweis von Tuberkelbacillen im NasenRachenschleim. Vielleicht sind diese Erscheinungen, die gewiss zum Teil auch von der Anstrengung, die eine so grosse Zahl von Untersuchungen verursacht, herrühren, der Ausdruck einer Bacillenwirkung.

Tabelle C und D enthalten die Aufstellung der Gesamtzahlen und Prozentsätze und sind zur Vervollständigung der Übersicht mitgeteilt.

Tabelle C. Zusammenstellung der Gesamtzahlen und der Prozentsätze.

\begin{tabular}{|c|c|c|c|c|c|}
\hline & 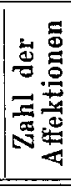 & 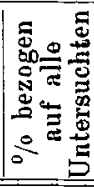 & & 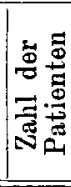 & 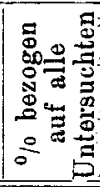 \\
\hline Gesamtzabl der Affektionen & 333 & $111 \%$ & Ohne pathol. Veränderungen & 73 & $24,3 \%$ \\
\hline
\end{tabular}

\begin{tabular}{|c|c|c|c|c|}
\hline & 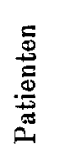 & $\begin{array}{l}\% \% \\
\text { von allen } \\
\text { Unter- } \\
\text { suchten }\end{array}$ & $\begin{array}{l}\% \text { v. Pat- } \\
\text { mit Affek. } \\
\text { tionen der } \\
\text { oberen } \\
\text { Luftwege }\end{array}$ & \\
\hline Nase allein erkrankt & 54 & $18 \%$ & $23,8 \%$ & $\begin{array}{c}=36,24 \% \text { d. untersuchten } \\
\text { Nasenkranken }\end{array}$ \\
\hline Rachen allein erkraukt & 37 & $12,3 \%$ & $16,3 \%$ & $\begin{array}{c}=32,2 \% \text { der untersuchten } \\
\text { Rachenkranken }\end{array}$ \\
\hline Kehlkopf allein erkrankt & 14 & $4,7 \%$ & $6,2^{\circ} ; 0$ & $\begin{array}{c}=20,3 \% \text { der untersuchten } \\
\text { Kehlkopflkranken }\end{array}$ \\
\hline $\begin{array}{l}\text { Nase und Rachen erkrankt } \\
\text { Nase, Rachen u. Kehlkopf } \\
\text { erkrankt }\end{array}$ & $\begin{array}{l}57 \\
19\end{array}$ & $\begin{array}{l}19 \% \\
6,3 \%\end{array}$ & $\begin{array}{l}25,2 \% \\
8,4 \%\end{array}$ & \\
\hline $\begin{array}{l}\text { Erkrankungen der oberen } \\
\text { Luftwege m it Beteiligung } \\
\text { der Nas } \theta \\
\text { Erkrankungen der oberen } \\
\text { Luftwege ohne Beteili- } \\
\text { gung der Nase }\end{array}$ & 55 & $18,5 \%$ & $\begin{array}{l}35,7 \% \\
24,2 \%\end{array}$ & \\
\hline
\end{tabular}


Tabelle D. Allgemeine Zusammenstellung.

\begin{tabular}{|c|c|c|c|}
\hline & & Zahl & Prozentsatz \\
\hline Nase: & $\begin{array}{l}\text { normal . . . . . } \\
\text { pathologisch . . . }\end{array}$ & $\begin{array}{l}151 \\
149\end{array}$ & $\begin{array}{l}\text { ca. } 50 \% \\
\text { ca. } 50 \%\end{array}$ \\
\hline Rachen: & $\begin{array}{l}\text { normal . . . . . } \\
\text { pathologisch . . . }\end{array}$ & $\begin{array}{l}185 \\
115\end{array}$ & $\begin{array}{l}61,7 \% \\
38,3 \%\end{array}$ \\
\hline Kehlkopf: & $\begin{array}{l}\text { normal . . } \\
\text { pathologisch } \\
.\end{array}$ & $\begin{array}{r}231 \\
69\end{array}$ & $\begin{array}{l}77 \% \\
23 \%\end{array}$ \\
\hline
\end{tabular}

So sehen wir denn, dass sowohl die grosse Zahl der gleichzeitigen Affektionen der oberen Luftwege bei Erkrankungen der Lunge als auch die an Menschen und Tieren gemachten Beobachtungen der letzten Jahre eine gewissenhafte Berücksichtigung und ein intensives Studium der genannten Teile gebieten. Noch wird es vorderhand nicht gelingen eine völlige Klärung der Verhältnisse und Einigung der weit auseinandergehenden Ansichten bedeutender Forscher zu erreichen. Bemühen wir uns daher, objektiv unsere Beobachtungen fortzusetzen und frei von Eigenliebe die Resultate anderer und unserer selbst zu prüfen und zu verwerten.

Aber schon erscheint in der Ferne das Bild eines alten Praktikers, der lange abseits rom Kampfe und nicht beeinflusst von dem überschätzten Werte einseitiger Forschung das Ergebnis seiner Untersuchungen am tuberkulösen Menschen auf sich einwirken liess. Unbekümmert um das Einzelgeplänkel lässt er das Hauptziel nicht aus den Augen und will sich nicht in den Spezialuntersuchungen verirren, die sich allzu weit vom Hauptwege verlieren. Die schon vor mehreren Jahren und auch erst kürzlich wieder geäusserte Ansicht (Münch. med. Wochenschrift Nr. 34) von Wolff-Immermann in Reiboldsgrün scheint ein einigendes Band um die Gegner schlingen zu wollen; mögen sie die primäre Affektion der Tuberkulose auch an noch so verschiedenen Gegenden des menschlichen Körpers annehmen, vom Wolffschen Standpunkte aus haben sie mehr oder weniger alle recht:

„Betrachten wir die Tuberkulose im Rahmen der gesamten Pathologie, so kommen wir dazu ein Bild von dem Wesen der Tuberkulose aufzustellen, das in wesentlichen Punkten von demjenigen abweicht, welches unter Führung der Bakteriologie in 
den letzten Jahrzehnten als das allein Zutreffende angesehen wurde. Wir sehen dann in der Tuberkulose eine chronischkonstitutionelle Infektionskrankheit, mit hämatogener Verbreitung in alle Organe; eine einmalige Infektion die zumeist in der Kindbeit. erfolgt, genügt zur späterer Erkrankung an Phthise, und eine zweite Infektion des einmal Infizierten bleibt eine oberflächliche Affektion ohne weitere Folgen" (Wolff).

\section{Therapeutischer Teil.}

Wenn ich meinen bisherigen Ausführungen noch einen die Behandlung betreffenden Absatz angliedere, so bin ich weit entfernt, die Therapie der vorher angeführten Affektionen auch nur in annähernd eingehender und vollständiger Weise zu besprechen; dieses dürfte wohl bei dem. Vorhandensein so vieler und ausgezeichneter Lehrbücher, unter denen mit Rücksicht auf die hier besprochenen Beziehungen der oberen Luftwege zueinander und zur Lungentuberkulose auch das vor kurzem erschienene von Schroeder und Blumenfeld erwähnt sei, ein überflüssiges Beginnen sein.

Ich möchte vielmehr über einige Beobachtungen berichten, welche ich an den Phthisikern unserer Anstalt gemacht habe, und die sich beziehen auf die

\section{Inhalationstherapie.}

Nachdem bekanntlich Traube die schon vor ibm von vielen Forschern angenommene Möglichkeit einer Wanderung von Staubteilchen bis in die Lungen durch exakte mikroskopische Untersuchungen klar bewiesen und G. Lew in an der Hand eines grossen statistischen Materials diese Beobachtungen gestützt hatte, glaubte man wohl annehmen zu dürfen, dass auf diesem natürlichen Wege eine rationelle Therapie der erkrankten Respirationsorgane sich bewirken liesse. Es wurden eine grosse Reihe von Untersuchungen gemacht, um festzustellen, wie sich die verschiedenen Arten des Aggregatzustand von Körpern auf dem Wege des normalen Luftstromes verhielten. War das Eindringen von trockenem Staube von Traube und Lewin bewiesen worden, so erhoben fein ausgedachte Experimente Waldenburgs die Annahme einer Einatmung zerstäubter Flüssigkeiten zur Tatsache; sie zeigten, dass feinste Partikelchen bis in die kleinsten Bronchien gelangen können, und dass in gleicher Weise die Möglichkeit des Eindringens von Dämpfen besteht. Das Ergebnis aller dieser Beobachtungen war die Tatsache, 
dass eine sowohl physikalisch als chemisch modifizierte Inspirationsluft bis in die tieferen Abschnitte des Atmungstraktus gebracht werden kann. Hierauf beruht der Ausbau der Inhalationstherapie, die sich in den einzelnen Fällen der Erkrankungen ebenso vieler Freunde auf der einen Seite wie Feinde auf der anderen erfreut! Ihren grossen Wert bei einer Reihe von Affektionen dürfte ihr mit Recht wohl niemand absprechen.

Neuerdings hat sie einen eifrigen Fürsprecher in Reitz (Leipziger Kongress für innere Med.) gefunden, der feststellte, dass mit Hilfe des von ihm konstruierten Zerstäubungsapparates Medikamente bis in die Alveolen gelangten. Er konnte die Resorption derselben durch den Nachweis der Mittel in Blut und Harn dartun. Obwohl dem von ihm vorgeschlagenen Wege der Lungenresorption zur Behandlung innerer Erkrankungen als dem natürlichen Resorptionsweg nicht gleichwertig in der Diskussion entgegengetreten wurde, so zeigte sich doch ein lebhafteres Interesse für die Inhalationstberapie als bisher. Vor allem tritt v. Schrötter nach dem Referate von G. Klemperer (Therap. d. Gegenwart 1904, Nr. 6) seit langem schon "für eine höhere Einschätzung" derselben ein. Er hat bei Hunden, welche durch den Bullingschen Apparat Methylenblau eingeatmet hatten bei der Sektion dasselbe bis in die feinsten Bronchien verteilt gefunden. D. Rotschild-Soden konnte Eisenchlorid in den Kavernen der Phthisiker nachweisen u. s. f.

Meines Erachtens ist bei richtiger Handhabung die Inhalationstherapie infolge ihrer auf natürlichem Wege ermöglichten Ausführung nicht nur die angenehmste für den Patienten, sondern auch die schonendste für die erkrankten Teile. Jede mit Hilfe instrumenteller Vorrichtungen vorgenommene Applikation, die sicherlich in einer grossen Zahl von Erkrankungen nicht umgangen werden kann, ist neben der medikamentösen Beeinflussung noch von anderen mit der Handhabung des Instrumentes verbundenen Wirkungen gefolgt, die in der grossen Mehrzahl der Fälle als unnötiger Reiz betrachtet werden müssen. Gewiss haften auch der Inhalationstherapie auf der anderen Seite eine Reihe von Unvollkommenheiten an. Ich möchte sie nicht für eine ideale Behandlungsmethode halten; jedoch auf ihre Vorzüge einzugehen und die Einwürfe ihrer Gegner zu besprechen, ist hier nicht der Ort.

Die Medikamente, welche in mannigfacher Form für die Einatmung angepriesen wurden, sind Legion und haben bei weitem sich nicht alle einen dauernden Platz in unserem Arzneischatze zu erringen vermocht. Um so gewagter mag es erscheinen, wenn ich wiederum ein Vittel zur Inhalation empfehle, das von Einhorn 
und Heinz entdeckte Nirvanin, welches mir allein als auch mit anderen Medikamenten gute Dienste geleistet hat.

Soweit mir die einschlägige Literatur bekannt ist, hat es bisher besonders in der Zahnheilkunde eine weiter verbreitete Anwendung gefunden, während die allgemeine Medizin in Deutschland sich weniger noch als in Frankreich mit ihm beschäftigte.

Nirranin ist das salzsaure Salz des Diäthylglykokollamidooxybenzoësäuremethylester und besitzt eine ausgesprochen anästhesierende Wirkung. Seine Vorzüge besonders vor dem viel angewandten Kokain sind folgende:

1. Es ist nach den Untersuchungen von Einhorn, Heinz, Luxemburger, Bonnard, Joanin, Reynier und meinen eigenen Beobachtungen sehr wenig giftig. Ich habe selbst die Maximaldosis, welche zehnmal grösser als die des Kokains, auf 0,5 angegeben worden ist, noch um ein Bedeutendes überschritten und nie üble Folgen gesehen.

2. Es besitzt antiseptische Kraft (Einhorn, Heinz, Luxemburger Sabrazès, Boisseau). Eine 1\% ige Lösung verhindert das Wachstum pathogener Mikroorganismen auf Gelatine, eine $t \%$ ige vermag sogar innerhalb sechs Stunden Milzbrandsporen abzutöten.

3. Auf dieser letzteren Eigenschaft beruht die schätzenswerte ziemlich lange Haltbarkeit im Gegensatze zu Kokain.

4. Zum Unterschiede von diesen lassen sich ferner die Nirvaninlösungen sterilisieren, ohne an ihrer Wirkung einzubüssen. Man kann sich also ein grösseres Quantum der Lösung vorrätig halten und einen Teil derselben vor dem Gebrauche sterilisieren.

5. Was die Anästhesie angeht, so ist sie ziemlich lange anhaltend und reichte bei den von mir vorgenommenen kleineren chirurgischen Eingriffen (Operationen von Unguis incarnat., Exstirpationen ron Hauttuberkulose, Inzisionen etc.) stets völlig aus. Wegen der mitgeteilten Eigenschaften des Mittels glaube ich ihm auch in der Inhalationstherapie einen grösseren Wert beimessen und mehr Beachtung wünschen zu dürfen, als es bisher besass. Ich habe es seit über einem Jahre an vielen Kranken erprobt, und möchte es auf Grund meiner Erfahrungen empfehlen.

Besonders gute Dienste leistete es mir bei solchen Patienten. welche im Anfangsstadium der Tuberkulose an dem sogenannten "Reizhusten" litten. Bekanntlich wird der "trockene Husten“, welcher den Lungenspitzenkatarrh so oft begleitet, sehr häufig vom Kehlkopfe oder Rachen ausgelöst, mag er in einem Katarrhe oder in chronischen Stauungszuständen seinen Grund finden. Durch Inhalation mit Nirvanin liess er sich jedenfalls in zahlreichen Fällen günstig beein- 
flussen und oft nach kürzerer Anwendung derselben dauernd beseitigen. Bei der Unschädlichkeit des Medikamentes ist es, obwohl sich auch mit den anderen Anästheticis vielleicht eine ähnliche Wirkung erzielen liesse, sicherlich diesem vorzuziehen. Ich habe selbst bei vier- bis fünfwöchentlicher täglich zweimaliger Inhalation von $15 \mathrm{ccm}$ einer $1 \%$ igen und auch beim gleichen Gebrauche einer $2 \%$ igen Lösung nie Unannehmlichkeiten beobachtet. Auch in den hartnäckigsten Fällen wurde eine wenn auch vorübergehende günstige Beeinflussung beobachtet. Wenn man die schädliche Wirkung einer ständigen Erschütterung der mehr oder weniger affizierten Lunge bedenkt, welche der für die Patienten sehr lästige Reizhusten im Gefolge hat, so wird man auch eine nur kürzere Beruhigung zu schätzen wissen.

Ich liess die Inhalation gewöhnlich 14 Tage lang (zweimal pro die $15 \mathrm{ccm}$ einer 1 - oder $2 \%$ igen Lösung) mit den allgemein verbreiteten kleineren Inhalationsmaschinen vornehmen und war dann auch meist in den Stand gesetzt, sie beiseite lassen zu können. Oft allerdings musste der infolge einer neuen Erkältung unserer empfindlichen Phthisiker rezidivierte Reiz wieder in der gleichen Weise behandelt werden. Dieser Umstand bedingt überhaupt gerade bei der Anwendung der Inhalationen in den Lungenheilstätten grosse Berücksichtigung. Die Einatmungen müssen mit Rücksicht auf die übrigen Kurverordnungen (Spaziergänge, Freiluft-Liegekuren etc.) zu einer solchen Stunde vorgenommen werden, dass der Patient sich nach derselben noch einige Zeit im Zimmer aufhalten kann, da sonst bei seiner grossen Disposition zu Erkältungen ihm die Behandlung des Halses eher schaden als nützen würde. Es empfiehlt sich ferner dieselben mit den mit Rücksicht auf den meist morgens und abends am stärksten auftretenden Husten zu dieser Zeit vornehmen zu lassen, jedoch nicht allzubald nach den Mahlzeiten.

Höher noch als die Unterdrückung des erwähnten lästigen Symptoms wird man den Wert des Nirvanins anschlagen in solchen Fällen, wo es gilt, in einem vorgerückteren Stadium der Tuberkulose schmerzhafte Affektionen zu behandeln. Unter diesen erwähne ich die einer erfolgreichen Therapie oft trotzenden recht starken Schluckbeschwerden, von denen manche chronisch Halskranke heimgesucht werden. Auch hier vermag das Mittel oft gute Dienste zu tun. Ich verweise zur Illustration auf fogenden in Kürze mitgeteilten Krankheitsfall, bei welchem uns das Medikament zu statten lkam.

Friedrich K., 56 Jahre alt, Wirt in Velbert, aufgenommen am 21. August 1903. Keine hereditäre Belastung. Seit März 1903 Husten, später Auswurf; Nachtschweisse und vorïbergehend Durch- 
fall, starke Abmagerung. Seit zwei Wochen Heiserkeit, die in den letzten Tagen von Schluckbeschwerden begleitet ist.

Diagnose: Beiderseitige Lungentuberkulose im Ir. Stadium, Kehlkopftuberkulose; Potatorium.

Die Schluckbeschwerden steigerten sich so sehr, dass selbst die eine Zeitlang genommene flüssige Nahrung vom Kranken verweigert wurde. Nach vergeblichen Versuchen das quälende Symptom zu beseitigen, griffen wir zur Nirvanininhalation. Ich brauche nicht zu betonen, dass wir von ihr nicht die Ausheilung der ausgebreiteten Larynxphthise erwarteten, vielmehr dem bereits als unheilbar prognostizierten Patienten nur Erleichterung verschaffen wollten. Dies gelang auch zu unserer Befriedigung. K., der nach der Hausordnung der Heilstätte als zum Aufenthalte in dieser nicht geeignet entlassen wurde, kehrte in die Behandlung seines Hausarztes zurück. Dieser bat uns nach kurzer Zeit mit dem Hinweis darauf, dass er bereits vieles zur Bekämpfung der Schluckbeschwerden versucht habe, um Mitteilung unserer Medikation, da der Kranke ihm erklärte, es habe ihm kein anderes Mittel solche Linderung verschafft, als das von uns angewandte.

Ich habe nach diesen noch einige ähnliche Fälle in der gleichen günstigen Weise beeinflussen können.

Bei der Ungiftigkeit des Nirvanins in geringen Dosen stehe ich nicht an, es auch gegen die kleineren Beschwerden unserer Kranken, wie Kratzen im Halse etc., welche sehr oft unbeachtet bleiben und in nicht seltenen Fällen zur Nervosität führen, zu verwenden; ich bin auch hier mit den Ergebnissen zufrieden.

In den letzten Monaten leistete mir eine Kombination von Nirvanin und Suprarenin gute Dienste, auf welche an dieser Stelle anch hingewiesen sei. Es kommt hierbei zu der anästhesierendè̀ Wirkung des ersteren noch die anämisierende des Nebennierenextraktes (ich möchte gleichzeitig die von $R$ osenberg (Therap. d. Gegenwart 1902 März) beobachtete Verringerung des Schluckschmerzes bei Angina durch Suprarenin erwähnen). Bei den chronischen Stauungszuständen der Schleimbäute, in deren Verlaufe es durch die ständige starke Füllung der Gefässe begreiflicherweise zu Erschlaffung der Muskulatur der Gefässwand kommen muss, erscheint mir die täglich mehrmals bewirkte Änderung des Gefässtonus durch Inhalationen von Nirvanin-Suprareninlösungen nicht ohne Wert. Jedenfalls möchte ich zum Versuche dieser Kombination aufzufordern mir gestatten; sie wurde von unsern Kranken gut vertragen.

Bei dieser Empfehlung des Nebennierenextraktes, dessen Herstellung wohl zu den besten Neuerungen im Gebiete der medikamen- 
tösen Behandlung der letzten Zeit zu rechnen ist, möchte ich die von mir angewandte Nasenspülung mit einer Lösung des Mittels nach operativen Eingriffen zur Sistierung von Blatungen erwähnen. Ich habe sowobl nach Entfernung von Schleimpolypen als auch nach Abtragung grösserer hypertrophischer Teile besonders der unteren Muscheln bisweilen gänzlich von einer nachfolgenden Tamponade $a b-$ sehen können und mich auf "Nasenduschen" beschränkt. Es gelang, die Blutung auch auf diese Weise ohne grosse Schwierigkeit zum Stehen zu bringen, ein Unstand, der von Patienten, deren Nase beiderseitig verstopft ist, sehr angenehm empfunden wird.

Wichtiger aber als dieses ist die auch von anderer Seite öfter betonte günstige Beeintlussung des Kokains durch das Suprarenin. Das in seiner Wirkung im einzelnen Falle so ganz und gar unberechenbare Anästhetikum ist ja nicht in allen Fällen za entbehren. Es dürfte aber feststehen, dass seine Giftigkeit bei gleichzeitiger Verwendung des Nebennierenpräparates sehr beschränkt wird. Ich habe stets zuerst Kokain appliziert und möglichst sofort hinterher Suprarenin nachgegeben. Eine Medikation, in deren Empfehlung ich mich mit Baeza-Haag (Berl. klin. Wochenschr. 1902 Nr. 52) eins finde, welcher es in dieser Anordnung zur Diagnosenstellung verwendet hat. Jedenfalls habe ich bei relativ grossen Kokaindosen mit erwähntem Zusatz nie üble Begleitexscheinungen an unsern Kranken gesehen.

Kehren wir nach dieser Abschweifung zur Inhalationstherapie zurück! Die vorher beschriebenen Inbalationen wurden, wie bemerkt, mit den bekannten kleinen Apparaten rorgenommen. Ich möchte nunmehr za der segensreichen Erfindtung der grösseren Vorrichtungen übergehen und deren Anwendung in unserer Heilstätte und ihr Ergebnis besprechen.

Seit einem halben Jahre besitzen wir den Wassmuthschen Apparat mit "Idealdampfzerstäuber und Reifscher Düse". Wie aus der beigegebenen Abbildung zu ersehen, fliesst die medikamentöse Flüssigkeit "vermöge ibrer eigenen Schwere von oben dem Zerstäuber $z u$ und wird dann an der äusseren Wandung derselben von der Druckluft in Empfang genommen und in feinster Verteilung der Raumluft einverleibt. Durch Regulierung der Pressluft sowie Flüssigkeitszufuhr kann man bestimmend auf die Zerstäubungsmasse, auf Zahl und Grösse der Tröpfchen wirken."

Nach Öffnung des Ventiles a fliesst der im Dampfreiniger gesärberte und in einem Wasserabscheider rom Kondenswasser befreite Dampf unter einem an dem Manometer $b$ ablesbaren and durch das Ventil a regulierten Drucke in den Heizkörper c, welcher durch die 
Schnur d mit der elektrischen Anlage verbunden ist. Der so ibberhitzte und trockene Dampf geht dann za dem Zerstäuber e, welchem. von oben durch ein Gummiröhrchen ans dem Gefässe f die medikamentöse Lösung zugeführt wird. Diese wird mit Hilfe des Dampfes durch die feinen Ottinungen der Dise $e$ in die Raumluft getrieben und kann nun ron den Kranken eingeatmet werden. Das in dem Wasserabscheider zurückbehaltene $\mathrm{H}_{2} \mathrm{O}$ fliesst durch ein Rohr in einen Kondenstopf, ron wo es abgeführt wird.

Unser Apparat, welcher schon mit einer Atmosphäre Druck gute

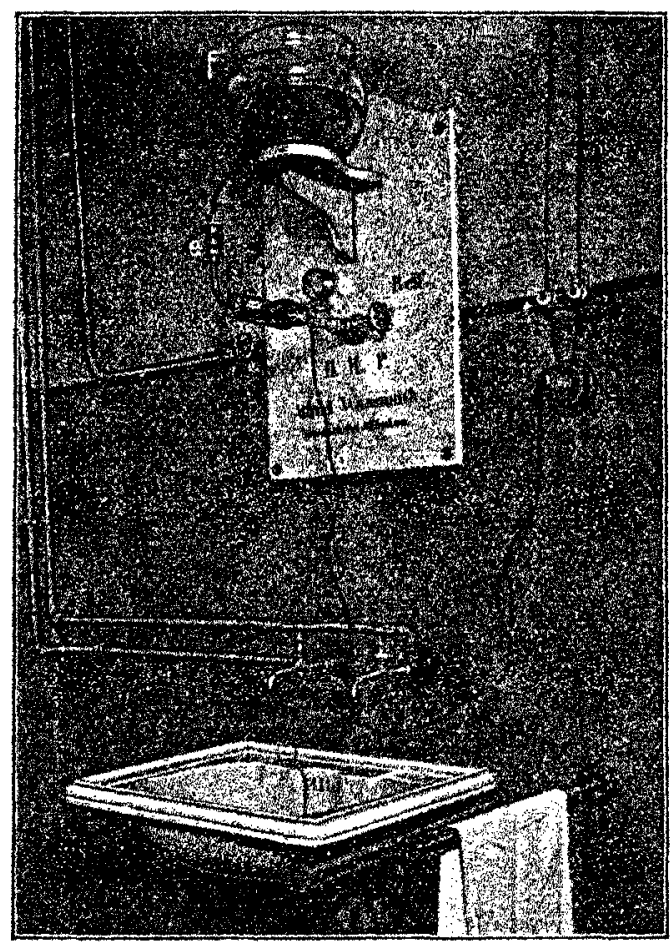

Abb. 1.

Resultate liefert, arbeitet mit 21/2 Atmosphären bisher $z$ unserer vollen Zufriedenheit. Was die Zerstäubung angeht, so verweise ich auf die Untersuchungen von Robinson (Dentsche medizin. Wochenschr. 1901) und Gerlach(Therap.Monatsbefte 1901), mit welchen unsere Beobachtungen übereinstimmen. Die zerstänbten Tröpfchen sind nngefähr von der Grösse der roten Blutkörperchen, zum Teil aber noch kleiner. Die besten Resultate zeitigt die Anlage, welche nicht mit Hilfe des gewöhnlichen nassen, sondern mittelst des überhitzten trockenen Dampfes die Zerstäubung bewerkstelligt, wie es auch

bei unserem Apparate der Fall ist.

Nach dem ron Wassmuth auf der XII. Jahresversammlung des allgemeinen Bäderverbandes (Balneolog. Zentralzeitung 1904 Nr. 9 und 10) gehaltenen Vortrage beträgt die Zahl der Tröpfchen bei Zerstäubung mit überhitztem Dampf pro qcm ca. 12970, die Grösse derselben ca. $0,001 \mathrm{~mm}$ im Durchmesser.

Bei der in der Heilstätte bereits vorhandenen elektrischen Anlage und Dampfheizung war die Aufstellung des beschriebenen Apparates nicht sonderlich schwierig. Aus der Damplleitung wurde die Press- 
luft durch ein Reinigungsfilter zugeleitet und durch einen mit Hilfe des elektrischen Stromes gebeizten Überhitzer getrocknet.

Die Einrichtung, welche für einen Raum von ca. $70 \mathrm{cbm}$ berechnet ist, kann mit verschiedenen medikamentösen Flüssigkeiten (selbstredend zu verschiedener Zeit) beschickt werden, and ermöglicht auf diese Weise eine Behandlung der einzelnen Affektionen der Luftwege mit den in Betracht kommenden Mitteln. Es können gleichzeitig eine Reihe von Kranken in den Inhalationsraum gelassen werden (in unserem Falle gewöhnlich $10-15$ ), und die mit dem Medikamente geschwängerte Luft atmen (Bild 2). Eine Schwester setzt den Apparat in Gang und fübrt die Aufsicht. Die Dauer der Einatmungen wechselt je nach Art des Medikamentes, welches benutzt wird; sie beträgt gewöhnlich $3 / 4$ Stunden.

Wie schon rorher bemerkt, sind wir mit der Einrichtung recht zufrieden; sie wird von den Kranken sehr geschätzt. Eine Raumbeschränkung findet durch die Aufstellurig des Apparates nicht statt, wie aus den Abbildungen leicht zu ersehen.

Wir lassen verschie-

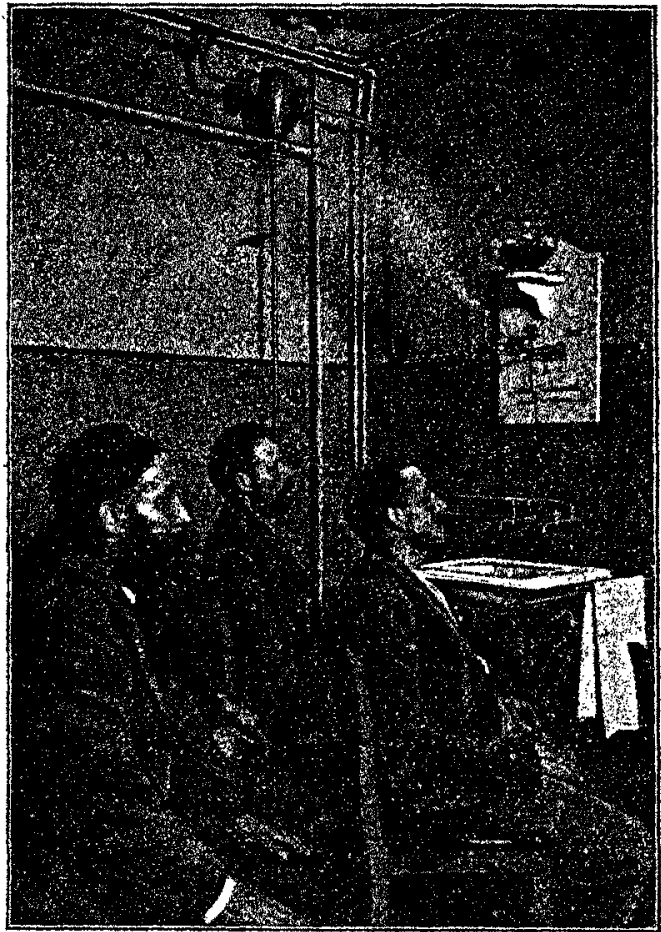

Abb. 2. dene Medikamente je nach der Erkrankung verstäuben. Ein grosser Teil der Patienten inhaliert das bewährte Emser Salz, ein anderer Reichenhaller Kiefern- und Latchenöl (Mack). Von den in Betracht kommenden adstringierenden Mitteln verwenden wir Alaun in 3\% iger Lösung, welches durch einen Zusatz von Nirvanin oder Tet. opii spl. auch für empfndliche Schleimhäute erträglich wird, in der letzten Zeit ferner Acidum tanmicum in 1 bis $2 \%$ iger Lösung, event. auch lombiniert mit Nirvanin (Bronchiektasien, Neigung za Blutungen etc.). Wir baben auch das von Bulling Münch. med. Wochenschr. $1904 \mathrm{Nr} .17$ ) empfohlene phenylpropionsaure Natron in einzelnen Füllen mit Hilfe der kleinen Apparate 
einatmen lassen, aber nicht die guten Erfolge gesehen, welche $\mathrm{B}$ ulling mitteilt. Ein endgültiges Urteil zu fällen dürften wir aber um so weniger in der Lage sein, da wir nicht den empfoblenen Thermovariator des Autors benutzen konnten.

Ein Hauptgrund, welcher mich abgesehen von dem mutmasslichen Frgebnisse der vorzunehmenden Untersuchungen zur Abfassung der vorliegenden Arbeit veranlasst, war die häufig gemachte Beobachtung, dass die Affektionen der oberen Luftwege im Beginne der Langentuberkulose besonders von den praktischen Ärzten allzuwenig berücksichtigt werden. Bei einer Umfrage erfährt man immer wieder, dass die Behandlung des Halses gar nicht oder in unzureichender Weise neben der Lungentherapie stattgefunden hat, selbst dann, wenn ausgesprochene Heiserkeit oder belegte Stimme die Aufmerksamkeit von vornherein auf die oberen Luftwege leitete.

In einem grossen Prozentsatze dieser Fälle ist aber eine zweckentsprechende Kur von Erfolg gekrönt, und kann für den Patienten mit Rücksicht anf die Komplikation von seiten der Pulmones recht Wertrolles leisten.

Es sollte aber auch die schon vorher erwähnte Gefahr der Descendenz der Erkrankung bei noch gesunder Lunge zu einer rationellen Behandlung der Anfangsstadien gemahnen. Man bedenke nur, welcher Vorteil den in staubigem Gewerbe beschäftigten Arbeitern erwacbsen wird, wenn man sie in der Lage erhält, die unreine Luft durch gesunde Organe zuträglicher zu machen oder wie Freudenthal sagt, zu "assimilieren". Welche immense Folgen die beruflichen Staubinbalationen zeitigen, zeigt die fleissige Dissertation von L. Spackeler-Berlin.

Die Heilstättenärzte haben die Pflicht, sich der Pflege der oberen Luftwege ganz besonders anzunehmen und event. vorhandenen oder vernachlässigten Erkrankungen derselben mit den zu Gebote stehenden Mitteln entgegenzuarbeiten. In einer Reihe ron Fällen wird die mildere Anwendung der Inbalation Genügendes zu leisten im stande sein; sie wird aber oft der instrumentellen und chirurgischen behandlung weichen müssen.

Darum müssen wir voll und ganz E. P. Friedrich zustimmen, der eine Anstalt, in welcher sich kein, erfahrener und technisch gewandter Kehlkopfarzt" befindet, zur Unterbringung ron Phthisikern für ungeeignet hält.

Herrn Chefarzt Dr. F. Köhler sage ich für die Überlassung des Materials und das Interesse, welches er meinen Untersuchungen entgegenbrachte, nochmals herzlichen Dank. 